Revue d'archéologie préhistorique

\title{
À propos de la variabilité technique et culturelle de l'entité gravettienne au Portugal : bilan des dernières découvertes et perspectives de recherche
}

Technical and cultural variability of the Portuguese Gravettian: recent

discoveries and research perspectives

Thierry Aubry, João Zilhão et Francisco Almeida

\section{(2) OpenEdition}

Journals

Édition électronique

URL : https://journals.openedition.org/paleo/508

DOI : $10.4000 /$ paleo. 508

ISSN : 2101-0420

Éditeur

SAMRA

Édition imprimée

Date de publication : 30 décembre 2007

Pagination : 53-72

ISSN : $1145-3370$

\section{Référence électronique}

Thierry Aubry, João Zilhão et Francisco Almeida, «À propos de la variabilité technique et culturelle de l'entité gravettienne au Portugal : bilan des dernières découvertes et perspectives de recherche », PALEO [En ligne], 19 | 2007, mis en ligne le 23 avril 2009, consulté le 25 juin 2021. URL : http:// journals.openedition.org/paleo/508; DOI : https://doi.org/10.4000/paleo.508

\section{(c)}

PALEO est mis à disposition selon les termes de la licence Creative Commons Attribution - Pas d'Utilisation Commerciale - Pas de Modification 4.0 International. 


\title{
À PROPOS DE LA VARIABILITÉ TECHNIQUE ET CULTURELLE DE L'ENTITÉ GRAVETTIENNE AU PORTUGAL : BILAN DES DERNIÈRES DÉCOUVERTES ET PERSPECTIVES DE RECHERCHE
}

\author{
Thierry AUBRY (1), João ZILHÃO ${ }^{(2)}$, Francisco ALMEIDA ${ }^{(3)}$
}

\begin{abstract}
Résumé : Nous proposons un bilan critique des données qui permettent d'organiser chronologiquement les faciès industriels connus, de caractériser les changements de choix techniques et de reconstituer l'environnement et les modalités de l'occupation gravettienne du Portugal. Cette analyse fondée sur des données géographiques, radiométriques, paléoenvironnementales, typo-technologiques, spatiales et anthropologiques cherche à évaluer les biais relatifs aux moyens mis en œuvre pour en établir les bases et le rôle des processus géologiques et pédologiques dans la préservation différentielle des sites et des vestiges archéologiques. En fonction de ce bilan, nous proposons des orientations de recherche susceptibles de pallier ces manques.

Mots-clés : Gravettien, paléoenvironnement, technique, industrie lithique, anthropologie, Portugal.

Abstract: Technical and cultural variability of the Portuguese Gravettian: recent discoveries and research perspectives.This paper presents a review of the evidence concerning the chronology of the different industrial facies, the nature of technological changes, the environment and the settlement patterns of the Portuguese Gravettian. Building on the geographical, radiochronometric, paleoenvironemental, typo-technological, spatial and human paleontological data, our analysis aims at an estimation of the role played and the biases introduced by geological and soil-weathering processes in the differential preservation of archaeological sites and remains. In conclusion, we suggest lines of research that might be able to overcome current shortcomings.
\end{abstract}

Key-words: Gravettian, Paleoenvironment, Technology, Lithics, Anthropology, Portugal.

\section{INTRODUCTION}

La thèse de J. Zilhão (Zilhão 1997) et ses différentes synthèses sur le sujet (Zilhão 1991a, 1991b, 1996, 2000, 2001 ; Zilhão et Almeida 2002) constituent le socle de toutes les recherches à venir sur le Paléolithique supérieur. Dans ces travaux, ont été définis le schéma chronostratigraphique et les différents " stades d'évolution" du Gravettien, définis sur des critères typo-technologiques, que nous réutiliserons ici. Les résultats obtenus ces dix dernières années ont permis de répondre à certaines des questions qui avaient été soulevées à l'occasion de ces travaux mais laissent cependant de vastes zones d'ombre, en particulier pour la phase initiale du techno-complexe gravettien.

\section{1- LES ACQUIS}

\section{1- Distribution des sites}

Si l'on reporte, sur la carte du Portugal, les occupations attribuées au Gravettien (fig. 1) on constate que leur distribution ne se confine plus aux seuls terrains sédimentaires, localisés entre le Tage et le Mondego. Néanmoins, les prospections systématiques effectuées dans le cadre de travaux de sauvetage ou programmés n'ont pas apporté la moisson de nouveaux sites que l'on pouvait légitiment espérer, dans les secteurs géographiques intermédiaires entre les concentrations qui se dessinaient (Zilhão 2001). En particulier, il n'existe toujours pas d'indice d'occupation gravettienne à proximité immédiate de quelques-uns des

(1) IGESPAR, IP - Extensão do IPA, largo da Piscina Municipal, 3100 - Pombal, Portugal - thaubry@sapo.pt (2) Department of Archaeology and Anthropology, University of Bristol, 43 Woodland Road, Bristol BS 8 1UU, UK joao.zilhao@bristol.ac.uk

(3) IGESPAR, IP - Avenida da India, 136, 1300-300, Lisboa, Portugal 


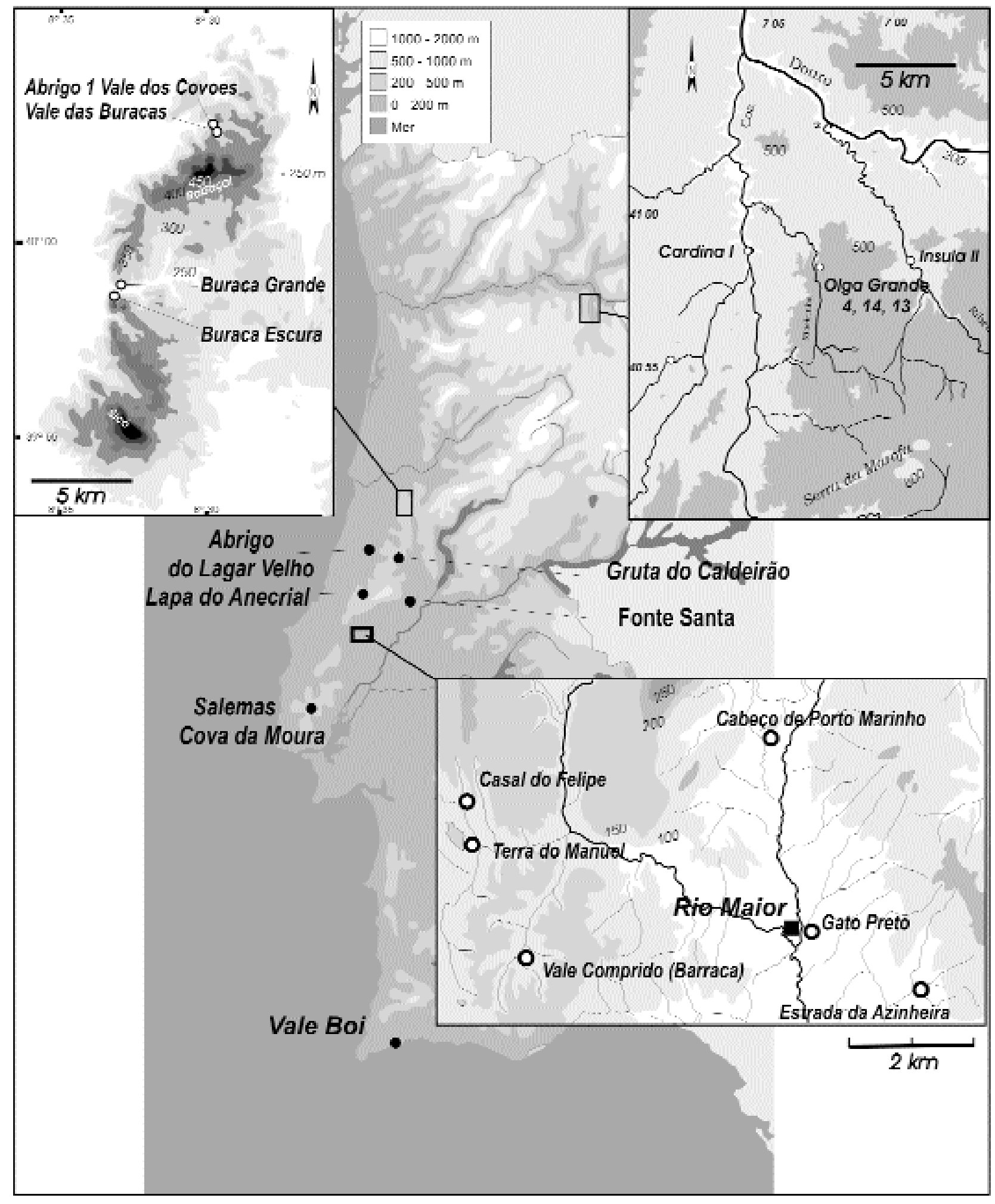

Figure 1 - Carte de localisation des sites gravettiens du Portugal.

Figure 1 - Location of Portuguese Gravettian sites. 
fleuves portugais (Mondego, Tejo, Guadiana), les sites de plein air connus dans leurs bassins se situant toujours sur des affluents de premier ou de second ordre et aucun indice d'occupation gravettienne n'a encore été détecté dans les domaines de moyenne et haute montagne.

La rive droite de la basse vallée du Mondego dont les silex du Jurassique ancien et moyen ont été déplacés vers les sites de la vallée du Côa (Aubry et al. 2001 ; Aubry et Mangado 2003), n'a livré qu'un seul indice possible d'une occupation gravettienne. La série lithique récoltée sur le site $n^{\circ} 46$ (inventaire des prospections de surface effectuées à proximité des sources de silex de la région de Cantanhede (Almeida et al. s.p.), présente des indices technologiques d'une production de supports lamellaires à profil rectiligne sur des nucléus à deux plans opposés de frappe lisses et inclinés, sur le silex local. Le choix préférentiel de cette modalité d'exploitation des nucléus semble être une des caractéristiques commune à plusieurs phases du Gravettien (Zilhão 1997).

La constatation d'un déficit en sites, dans plusieurs régions dont la fréquentation est attestée au moins pour la collecte de silex, pose clairement le problème de la représentativité du registre archéologique disponible. En effet, on manque encore de données technologiques fiables pour pouvoir caractériser chronologiquement des ensembles lithiques récoltés en surface sur des sites de plein air localisés à proximité des sources de matériaux siliceux. Par ailleurs, les données obtenues sur la séquence d'occupation du site de Fariseu dans la vallée du Côa (Aubry et al. 2002) montrent la difficulté de repérage des occupations humaines conservées en limite des plaines alluviales et des versants de ces cours d'eau, où les phénomènes érosifs ont été particulièrement marqués à la fin du Pléistocène et où le taux de sédimentation alluviale a fortement augmenté depuis le MoyenÂge (Zilhão 1997b ; Aubry 2002).

La carte de distribution des sites (fig. 1) indique que la majorité reste encore localisée à proximité des affleurements de silex du Cénomanien de la région de Rio Maior, suivie par plusieurs groupements de moindre densité. La découverte récente de niveaux d'occupation gravettiens au sein de la séquence de l'abri de Vale Boi (Bicho 2004) fournit un indice d'occupation en limite occidentale de l'Algarve et comble un vide entre les occupations du centre du Portugal et celles de la côte méditerranéenne espagnole.

Les espaces géographiques concernés sont situés surtout dans l'auréole sédimentaire de l'ouest de la Péninsule ibérique mais comprennent aussi les terrains du socle paléozoïque en bordure occidentale de la Meseta. Dans les grottes et abris des massifs calcaires périphériques, ils s'étalent entre 160 (Casa da Moura) et 540 mètres, si l'on accepte l'attribution gravettienne proposée pour les couches I et $J$ de la grotte de Lapa do Picareiro malgré les résultats obtenus pour des datations sur charbons (Bicho et al. 2003) ; dans les vallées et plateaux du Côa et de la haute vallée du Douro portugais, ils s'étalent entre 160 (Cardina I) et 550 mètres d'altitude (Olga Grande 4 et 14).

\section{2 - La séquence chronostratigraphique}

1.2.1 - Les datations absolues et les problèmes de conservation différentielle

De nombreuses dates ont été obtenues à partir d'os et de charbons, par le procédé ASM, sur les séquences de l'abri de Lagar Velho (Pettit et al. 2003 ; Zilhão et Almeida 2003 ; Almeida 2003), de la grotte de Buraca Escura (Aubry et al. 2001), de la grotte de Caldeirão (Zilhão 1997), de la grotte de Anecrial (Zilhão 1997 ; Almeida et al. 2004) et de Vale Boi (Bicho et al. 2004) et par le procédé conventionnel, sur les séquences de Cabeço de Porto Marinho (Zilhão et al. 1995 ; Zilhão 1997). Les sites de plein air de Cardina I et Olga Grande 4 ont fait l'objet de datations par le procédé TL (Mercier et al. 2001 ; Valladas et al. 2001), ce qui est aussi le cas du site de plein air de Vale Comprido (Cruzamento) (Zilhão 1997).

La phase finale continue à être la mieux datée et les lacunes concernent les moments antérieurs de la séquence du Gravettien. L'analyse des données fait apparaître que celles qui concernent les phases antérieures de la séquence gravettienne sont probablement directement liées à des érosions. Ces demières n'auraient pas permis la conservation des macro restes organiques qui ne se seraient pas conservés par manque de recouvrement (Zilhão et Almeida 2002). La concentration des dates radiocarbones en trois groupes serait alors en relation avec les phases à plus fort taux de sédimentation de faible énergie, mises en évidence à Lagar Velho (Angelucci 2002, 2003). Ils sont situées respectivement autours de 27 000/29 000, 24 500/25 000 et 21 500/23 000 BP (Zilhão et Almeida 2002, p. 55), et le premier et le dernier de ces trois moments de sédimentation pourraient correspondre à des évènements de Heinrich (HE2 et HE3). Pour d'autres auteurs, les lacunes observées seraient la conséquence directe des fluctuations de la fréquentation humaine et des variations de densité de population (Marks 2000).

Pour la période comprise entre 27000 et 25000 BP on ne dispose que de deux dates radiocarbones. Dans la séquence de la grotte de Caldeirão, un résultat de 26020 \pm 320 BP (OxA-5542) a été obtenu par le procédé ASM sur une esquille d'os de la couche $\mathrm{Jb}$, laquelle n'a pas foum $\mathrm{i}$ de matériel diagnostique dans la surface fouillée. Un résultat de $26560 \pm 450$ BP (GifA-97258) a été obtenu par le même procédé sur un fragment de vertèbre de bouquetin provenant de la base de l'unité stratigraphique $2 f$ de la grotte de Buraca Escura (Aubry et al. 2001), en association stratigraphique avec une extrémité de pointe à retouche bilatérale directe (fig. 2). Cependant, il faut prendre en compte qu'un évènement érosif en relation avec une régression marine antérieure à $27100 \pm 900 \mathrm{BP}(\mathrm{OxA}-$ 10849) tronqua les ensembles $B s$ et $A l$ de la séquence de Lagar Velho (Angelucci 2002, 2003) et le fait que cet épisode soit suivi d'une phase de stabilisation qui pourrait atteindre plusieurs millénaires demande à ce que la contemporanéité entre l'élément daté et la pièce archéologique de la couche $2 f$ de Buraca Escura soit confimée (Zilhão et Almeida 2002). 


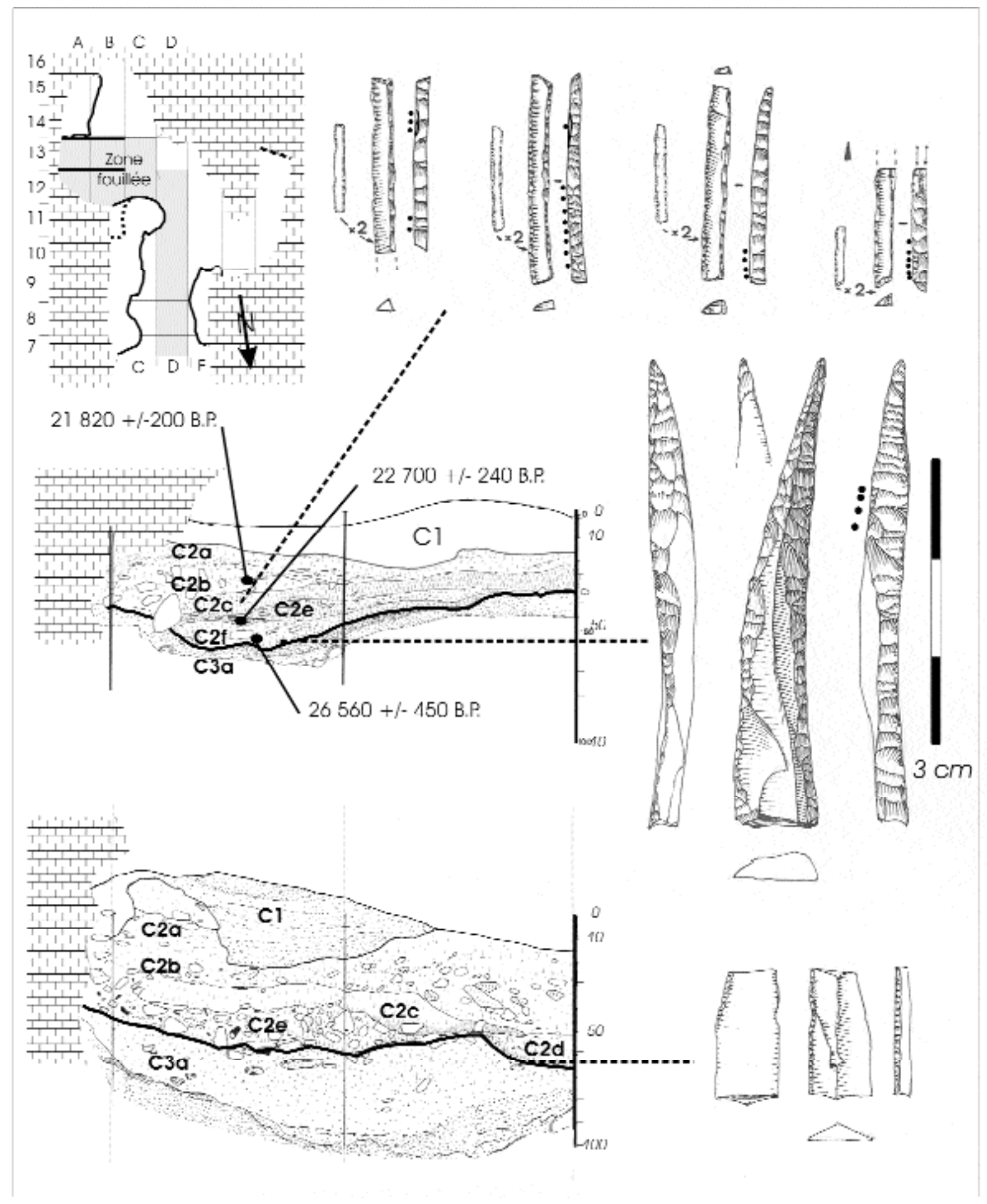

Figure 2 - Buraca Escura, Coupes stratigraphiques, position des éléments datés et d'armatures retouchées.

Figure 2 - Buraca Escura cave: stratigraphic profile with the location of the samples dated and a selection of retouched bladelet. 


\subsection{2 - Evolution diachronique des outillages}

Excepté les fragments de sagaies encore inédites des couches gravettiennes du site de Vale Boi (Bicho et al. 2003) aucun élément d'industrie osseuse n'a été jusqu'à présent récolté dans des conditions stratigraphiques permettant une association sûre avec un élément daté. Néanmoins deux pointes en os de la grotte de Casa da Moura (Zilhão 1997, p. 189, Roche 1951) et un os pénien de la grotte de Salemas (Zilhão 1997, p. 483 ; Roche et a l. 1962) se rapprochent des pointes en os dites d'Isturitz qui sont traditionnellement associées à la phase moyenne du Gravettien, à burin de Noailles (Bricker 1995 ; Goutas 2004). Les industries lithiques constituent donc la seule base dont nous disposons pour établir la séquence chronostratigraphique.

Les séries gravettiennes du Portugal se caractérisent toutes par l'utilisation de galets de quartz et de quartzite pour la production d'éclats qui sont utilisés bruts ou pour la confection d'outils sur éclat. On rencontre ces productions, aussi bien là où des silex sont disponibles à proximité du site d'abandon, que dans des contextes où aucune ressource siliceuse n'existe à proximité (Zilhão 1997 ; Almeida et al. 2002 ; Almeida 2003 ; Thacker 1996 ; Bicho et al. 2003 ; Aubry et Sampaio 2003a, 2003b, Aubry et Mangado 2003). Dans les sites de la vallée du Côa, la disponibilité locale en ressources lithiques permettant l'obtention d'éclats massifs semble avoir dicté l'utilisation du quartz pour la confection de grattoirs larges sur éclats, pendant plusieurs phases du Gravettien. De même, si dans ces sites l'utilisation du silex, de variétés de silices hydrothermales et du cristal de roche est dominante pour la confection de lamelles à dos et de pointes à dos, le quartz a parfois été utilisé comme support pour ces types d'armatures.

La quantification des différents types de matériaux lithiques utilisés au long du Gravettien met en évidence un choix manifeste du quartz pendant la phase finale, même pour des sites localisés à proximité de sources de silex d'excellente qualité (Marks et Almeida 1996 ; Zilhão 1997 ; Almeida 2000 ; Aubry et al. 2001)

L'étude des techniques de retouche et de production des supports de l'outillage retouché sur support lamellaire n'a pas encore été abordée de manière systématique et selon les mêmes critères d'analyse pour les séries lithiques de cette période ; toutefois, les études disponibles permettent de définir plusieurs procédés de production lamellaire (fig. 3) :

- une production de lamelles, selon une progression frontale, tournante ou semi-tournante, à carène et cintre peu marqué sur des nucléus prismatiques à un ou deux plans de frappe opposés, à partir de nodules de silex, des cristaux de quartz hyalin, des fragments de roches filoniennes ou des galets de roches siliceuses à grain fin (fig. $3, n^{\circ} 3-$ 8). Par ailleurs, un procédé original consistant à préparer une crête à l'intersection d'enlèvements effectués depuis le dos des nucléus est attesté pour le quartz et le cristal de roche (fig. $3, n^{\circ} 1$ et 2 ) ;
- une production de lamelles sur des éclats, utilisant une fracture ou une troncature comme plan de frappe (type burin sur troncature) parfois à deux plans de frappe opposés (fig. 3, $n^{\circ} 13-15$ ) ;

- une production de lamelles sur des blocs ou des éclats aboutissant à des nucléus carénés (fig. $3, n^{\circ} 9$ ) ;

- une production d'esquilles et de lamelles par percussion posée sur enclume. Les supports utilisés sont alors des éclats, petits nodules, cristaux de quartz, ou des nucléus préalablement exploités selon les procédés précédents (fig. 3, $\mathrm{n}^{\circ}$ 10-12).

Le choix entre ces différents procédés, leur relation avec la distance des sources lithiques exploitées et la fonction des sites au long de la séquence gravettienne dépassent le cadre de cette synthèse. Néanmoins, l'étude des armatures sur lamelles retouchées permet de définir plusieurs groupes possédant des caractères communs et de rares séquences stratigraphiques fournissent des arguments pour en définir la chronologie et la position relative.

Plusieurs catégories typologiques de pointes à dos et de procédés de production des supports lamellaires (fig. 3 et 4) sont associées dans l'unité stratigraphique 3 du site de Olga Grande 4. L'analyse spatiale (cf. chapitre 1-3) indique une constitution " rapide " lors d'un court passage (Aubry 1998, 2001,2002 ) et confirme ainsi la diversité qui apparaissait sur les assemblages déjà connus, récoltés dans des conditions et milieux ne permettant pas d'évaluer la durée de formation, comme à la grotte de Salemas (Zilhão 1997). Néanmoins, les cinq datations obtenues sur des fragments de quartzite provenant d'un foyer à remplissage pierreux de la base de l'unité 3 de Olga Grande 4, sont comprises entre 26800 et 31000 BP, avec une large marge d'erreur (Mercier et al. 2001, Valladas et al. 2001) et ne permettent pas de lui associer une chronologie précise (la comparaison avec les résultats radiocarbones doit aussi prendre en compte le fait que cette dernière méthode sous-estime de façon significative l'âge réel, calendaire, des échantillons, tel qu'il est exprimé par les résultats TL).

La sur-représentation des nucléus obtenus par percussion posée sur enclume, en silex et cristal de roche, comparativement aux esquilles et lamelles produites par ce procédé ne peut pas être seulement expliquée par un tri géologique. Une étude en cours (travail en collaboration avec $L$. Klaric, basée sur un référentiel expérimental) pourrait confimer qu'une partie de ces esquilles auraient été destinées à être montées brutes (ou légèrement transformées ?), comme l'a proposé Zilhão (1997), à partir de la fréquence de représentation des "pièces esquillées " au long du Paléolithique supérieur.

Cette phase d'occupation caractérisée à Olga Grande 4, serait antérieure à celle caractérisée par une forte proportion de microgravettes (lamelles étroites à dos abrupt obtenu par retouche croisée, ou plus rarement directe, appointée, dont la base est fréquemment aménagée par une retouche inverse) et de lamelles à dos, où les éléments 

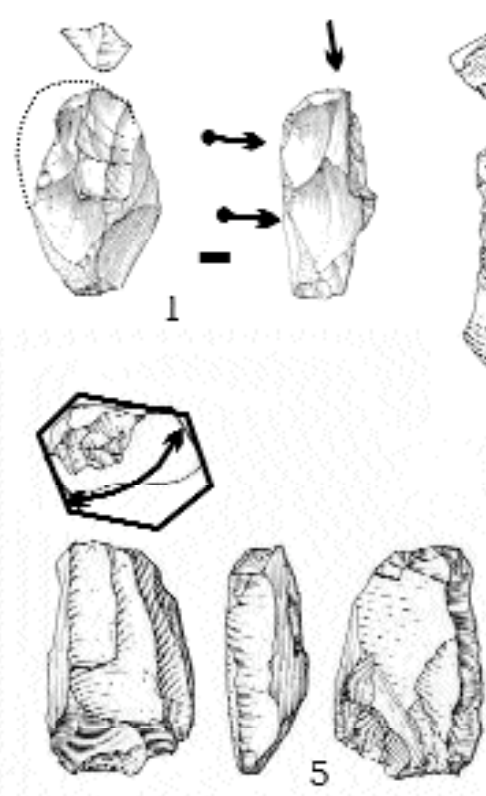

5
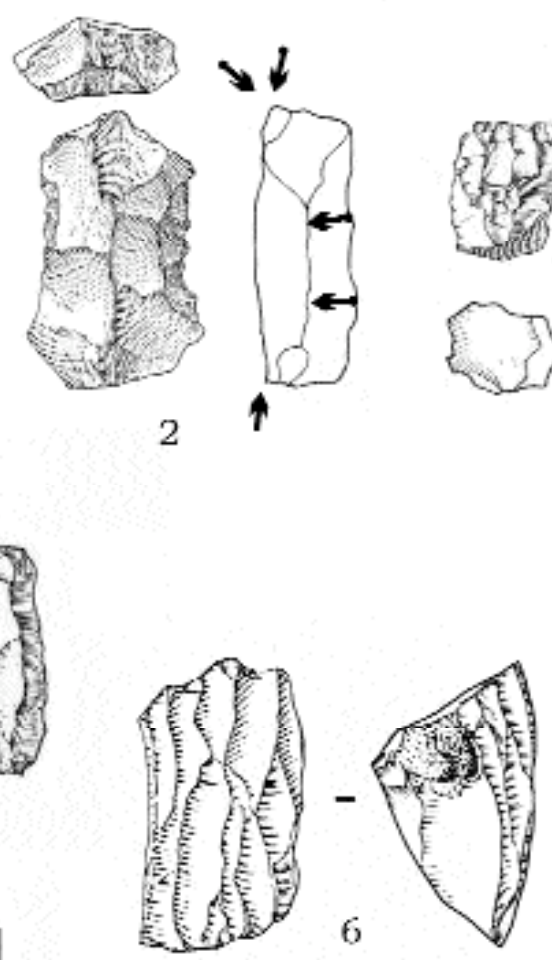

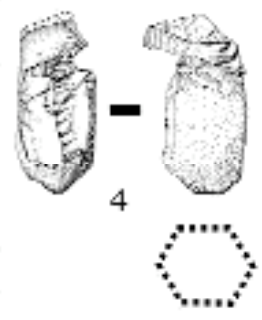

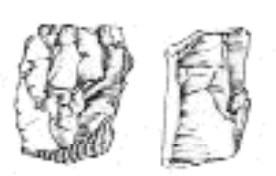

3

$\{3\}$

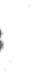

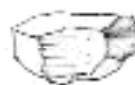
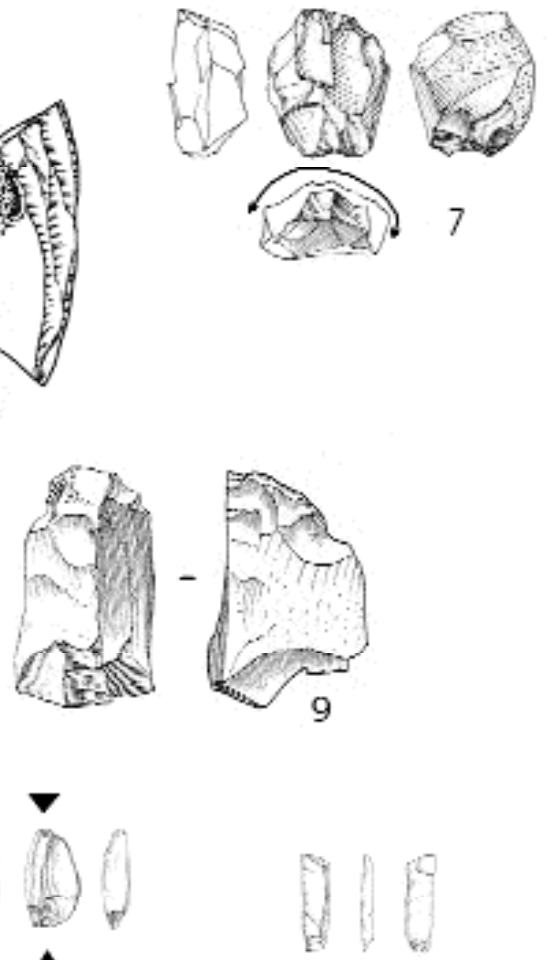

12

10

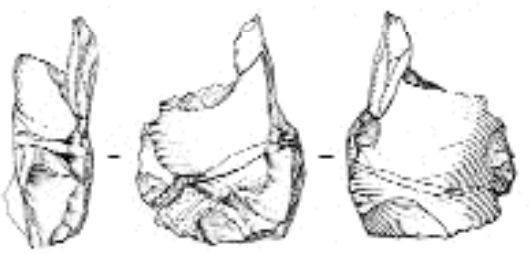

13
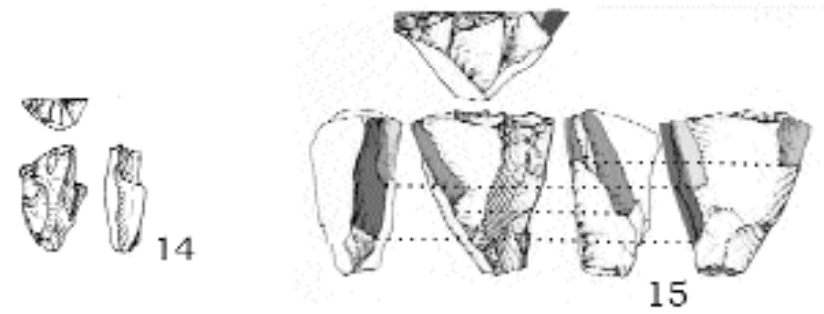

Figure 3 - Méthodes de production de support lamellaires reconnus sur des séries lithiques gravettiennes, 1 et 4 : Olga Grande 14, UE3 base, 1, 3, 11, et 12 : Cardina I, UE4 UA 10, 5, 8 et 13 : Olga Grande UE3 base, 6 : Cabeço de Porto Marinho II, niveau inférieur, 7 et 14 : Insula II UE 2, 9 : Lapa de Anecrial c2, 15 : Abri de Vale dos Covões, UE5.

Figure 3 - Bladelet core reduction sequences represented in lithic assemblages of the Portuguese Gravettian. 1 and 4: Olga Grande 14, UE3 base. 1, 3, 11 and 12: Cardina I, UE4 UA 10. 5, 8 and 13: Olga Grande UE3 base. 6: Cabeço de Porto Marinho II, lower level. 7 and 14: Insula II UE 2. 9: Lapa de Anecrial, level 2. 15: Vale dos Covões shelter, UE5. 


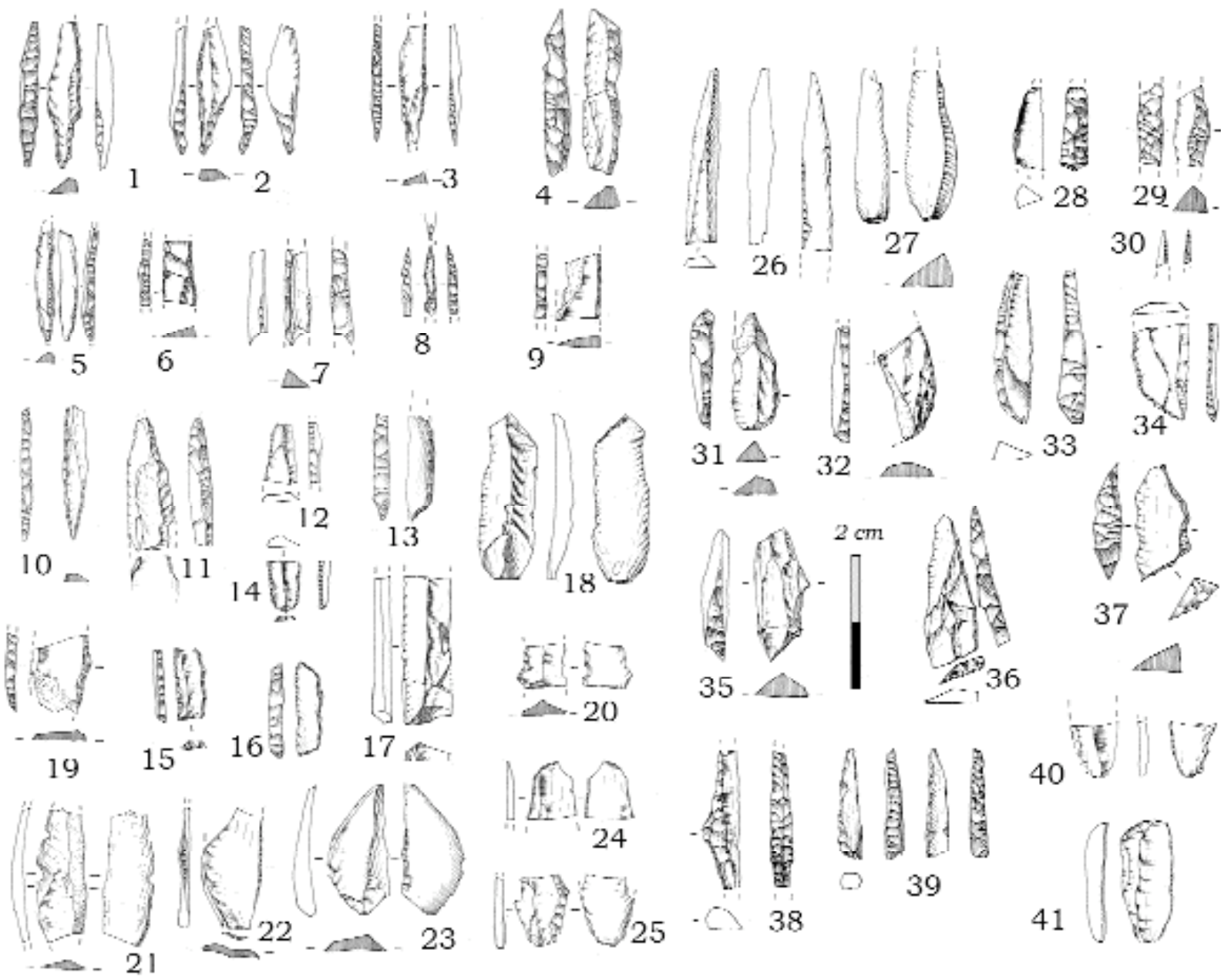

Figure 4 - Olga Grande, unité stratigraphique 3, armatures retouchées sur support lamellaires.

Figure 4 - Olga Grande, level 3. Bladelet tools.

géométriques et gravettes à base tronquée sont absents. En effet, la séquence qui a été établie récemment lors des fouilles dans l'abri 1 de Vale dos Covões (fig. 1 et 5), montre une composition distincte des catégories typologiques de pointes à dos au long d'une séquence que nous interprétons comme gravettienne (Aubry et al. en préparation) malgré les résultats d'âge tardiglaciaire obtenus (par le procédé ASM sur un charbon du sommet de la couche 5 , et conventionnel sur des charbons de la base de la couche $8 b$ ), que nous pensons être plutôt en relation avec les occupations magdaléniennes sus-jacentes (fig. 5, U.E. 7 à 5).

La phase représentée dans l'unité 5 montre des microgravettes typiques un schéma de production de supports lamellaires sur des nucléus/burins sur troncature voisin de celui observé en France (fig. 3), pour le Gravettien récent de la base de la séquence de l'abri du Blot (Klaric 2000 et 2003) et du Périgordien VI de la séquence du Sud-Ouest aquitain (Bricker et al. 1995). Une fonction de nucléus pour la production de supports lamellaires pour armatures microlithiques (des microgravettes, des pointes à dos courbe et lamelles à dos), avait déjà été proposée pour les burins sur troncature de Vale Comprido (Barraca). Ce gisement de plein air situé à côté de celui de Vale Comprido (Cruzamento), fouillé avec des méthodes modernes, n'en est en toute probabilité qu'une zone marginale épargnée par les fouilles anciennes (Zilhão 1997). Vu le résultat TL obtenu pour Vale Comprido (Cruzamento) (27 $900 \pm 2200$ $\mathrm{BP}$ ), et compte tenu de la sous-estimation (trois à quatre millénaires, pour l'époque en question) de l'âge radiocarbone, ce Gravettien portugais à burins sur troncature correspondrait à une phase récente du Gravettien.

Les phases postérieures du Gravettien final se placent entre 22500 et 21500 BP (Zilhão (1997) et Almeida (2000) ce que semble bien confirmer les résultats obtenus à partir de l'analyse technologique de l'ensemble TP06 de la séquence de l'abri de Lagar Velho (Almeida et al. 2003), et 

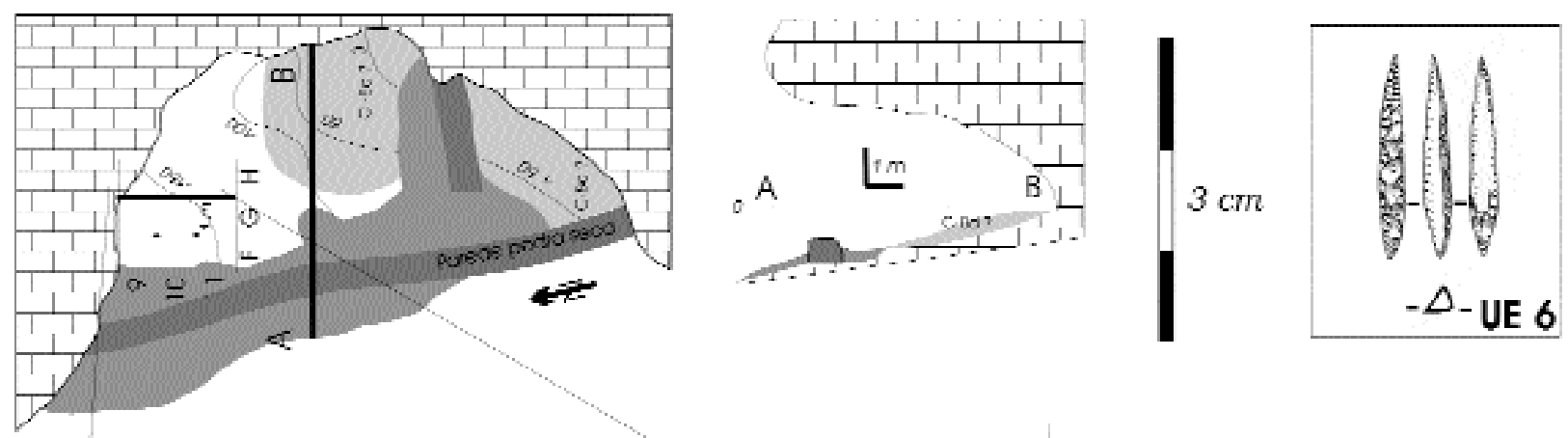

\section{Abrigo de Vale dos Covões}

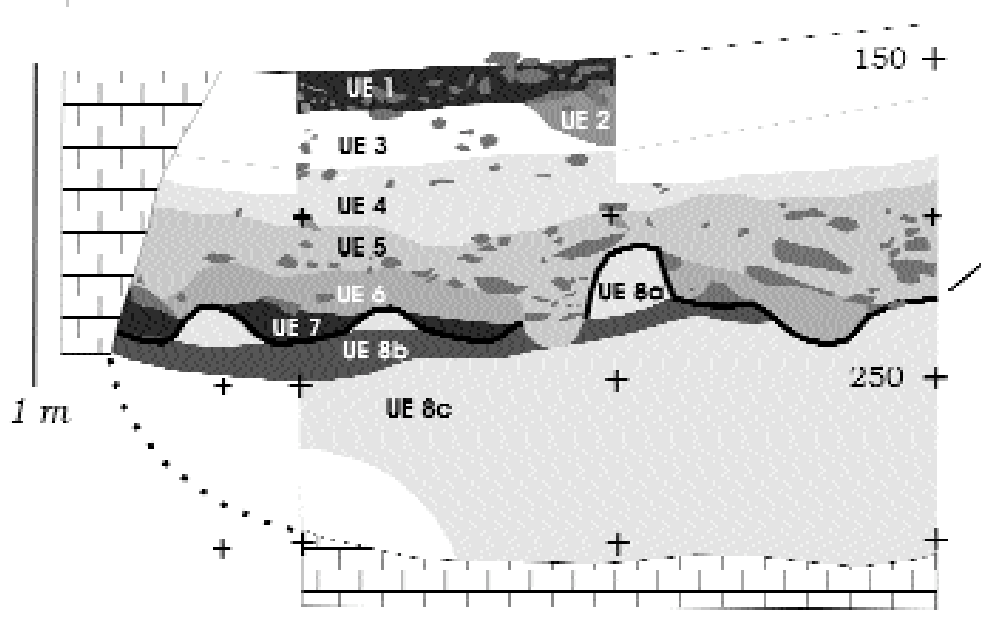

$+$

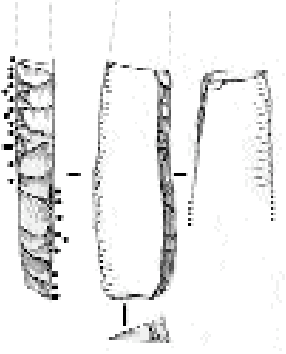

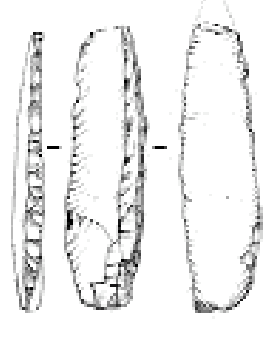

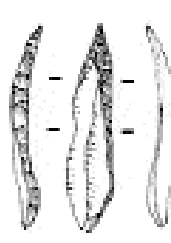

UE 8b base

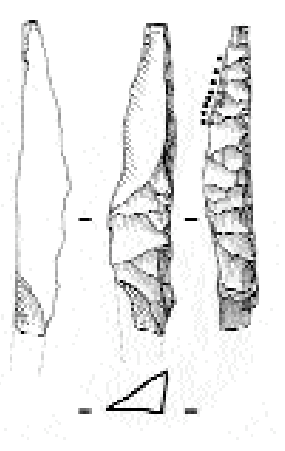

Figure 5 - Abri de Vale dos Covões, coupe stratigraphique de référence et sélection d'armatures retouchées sur supports lamellaires.

Figure 5 - Vale dos Covões. Stratigraphical profile and a sample of retouched bladelets from the different levels. 


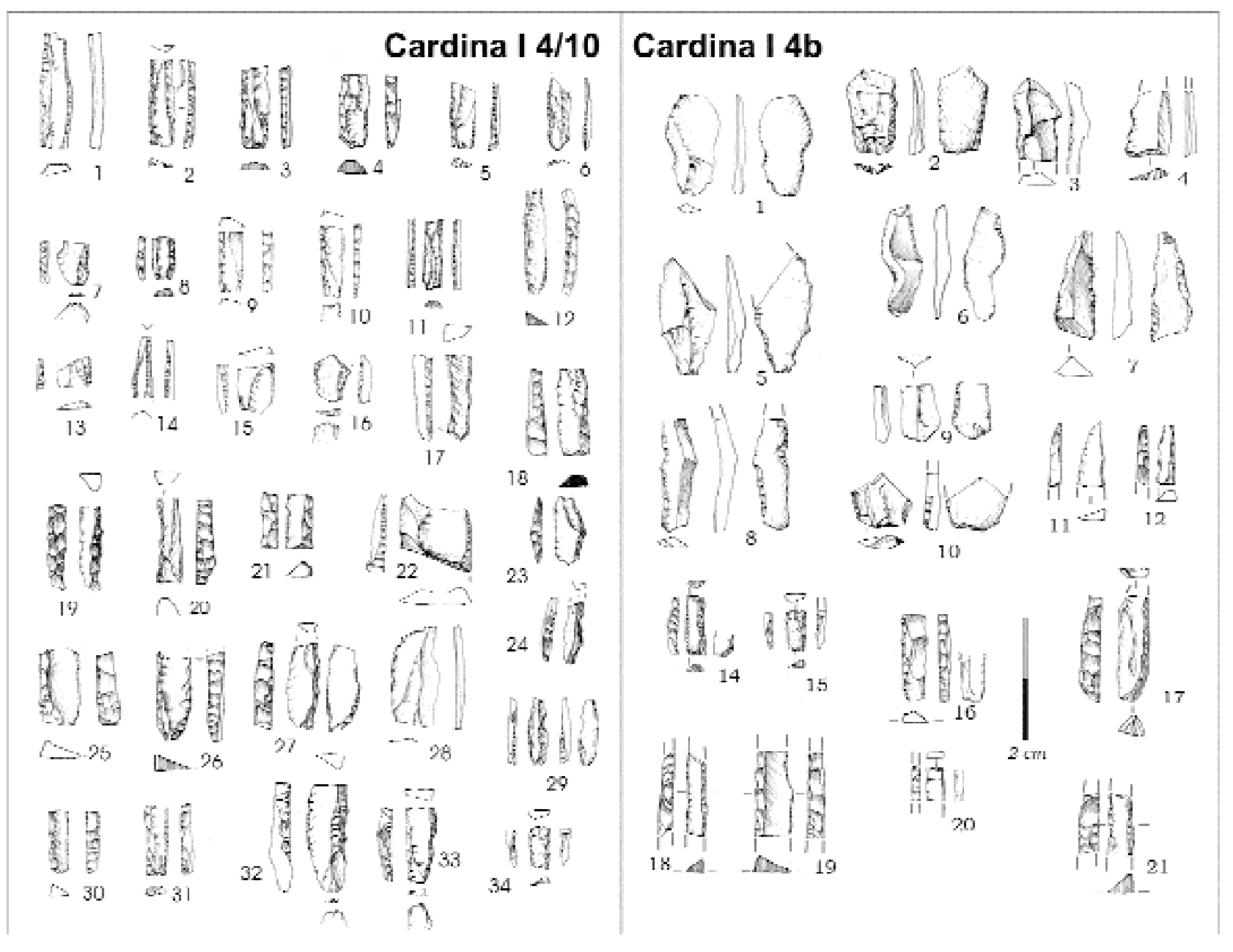

Figure 6 - Cardina l, armatures retouchées sur support lamellaires, unités stratigraphique 4/10 et 4b.

Figure 6 - Cardina I. Typology of bladelet tools in levels 4/10 and 4b.

de l'étude typo-technologique des riches ensembles lithiques provenant des unités $4 \mathrm{~b}$ et 4 base du site de plein air de Cardina I (Aubry et al. 2003c, fig. 6). Sur ce site de plein air, l'analyse des supports lamellaires et des procédés de retouche des lamelles à dos et à dos tronqué, de l'unité artificielle 10 de la couche 4, permet d'observer que l'ampleur de la réduction de largeur liée à la confection du dos par retouche est moindre que pour les exemplaires de la même catégorie typologique provenant du sommet de la couche 2 e de la séquence de Buraca Escura (fig. 2). La faible représentation des armatures de ce type dans la couche sous-jacente $4 \mathrm{~b}$ (fig. 6, niveau 4b) ne permet pas de donner une signification chronologique à ce détail, qui demanderait à être confirmé sur d'autres séquences.

Les procédés de productions des supports lamellaires sont semblables à ceux représentés à Olga Grande, où s'ajoute la production de lamelles sur des fronts de grattoirs carénés (fig. 3). A Cardina I, le déficit en esquilles et lamelles produites pas percussion posée est attesté sur des silex allochtones provenant de plus de $150 \mathrm{~km}$. Le taux maximum de fragmentation, aboutissant à des nucléus de moins d'un centimètre, n'est possible que par cette méthode et ne peut s'expliquer par un souci d'économie puisque ce type de production est attesté sur le silex et le quartz pour des sites où ces matériaux sont d'origine locale (Aubry et al. 2001 ; Almeida 2003, et s.p.).

La présence de quelques burins sur des supports de petit gabarit qui évoquent le type "Noailles" dans les unités $4 \mathrm{~b}$ et 4/10 du site de Cardina I, associés à des lamelles à dos tronquées est à noter (fig. 7). Nous ne possédons pas assez de données pour trancher entre une convergence de forme ou un rapprochement peu probable avec les assemblages de nombreux burins de Noailles, plus anciens, en France et dans le nord de l'Espagne. 


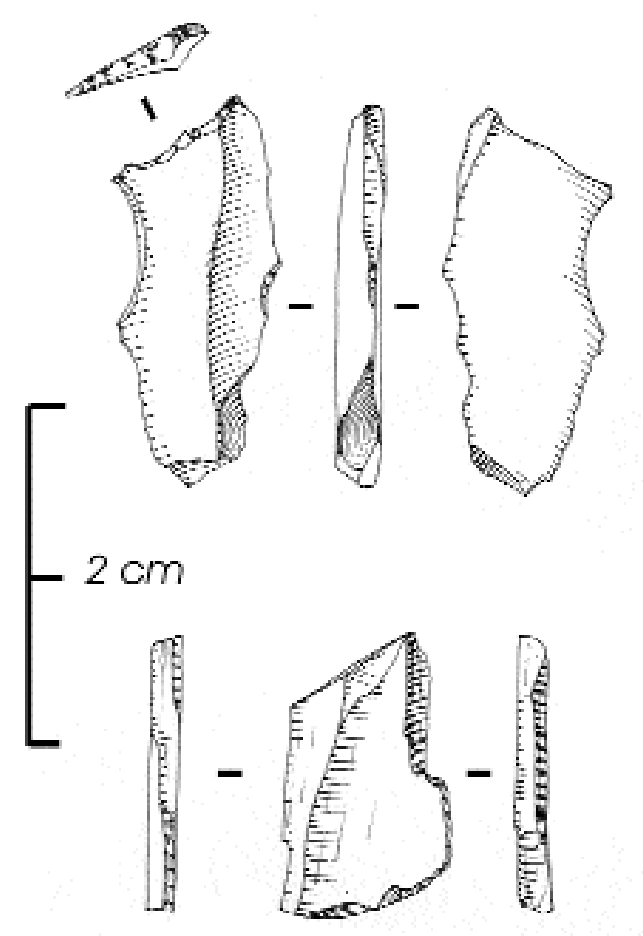

La période chronologique entre 25000 et 21500 BP commence donc lentement à être balisée de points d'ancrages et plusieurs études typo-technologiques sont en cours. Cependant, aucune nouvelle donnée ne permet de savoir si l'on doit continuer à y placer, sur la seule base de la présence de pointes à retouche abrupte, la phase du Fontesantense, définie à partir des séries de Fonte Santa (trois dates obtenues par le procédé TL sur des silex chauffés, comprises entre $35300 \pm 3600$ BP et $40400 \pm$ 4600 BP) et de Casal de Felipe. De même, il reste à établir la chronologie de l'assemblage lithique comprenant des lamelles à retouches marginales, reconnues sous des niveaux contenant des vestiges solutréens, à la base de la séquence du site du versant de Vale das Buracas (Almeida et Neves 2003 ; Almeida et al. 2004).

\section{3 - L'analyse spatiale}

L'approche synchronique est probablement celle où les études menées à partir des séries découvertes sur des sites fouillés selon des méthodes modernes ont permis d'obtenir des résultats des plus novateurs concernant l'organisation spatiale de niveaux d'occupation et la restitur tion des comportements des gravettiens. En effet, les résultats obtenus à partir de l'analyse spatiale de la distribution et des relations spatiales fondées sur les remontages lithiques mettent en évidence des moments où la conservation post-dépositionnelle des vestiges abandonnés sur des sols d'occupation du Gravettien final est bonne, relativement à d'autres régions d'Europe où les processus liés aux alternances du gel-dégel ont eu un impact important. L'absence de phénomènes de déplacements post-dépositionnels de grande ampleur, tant en milieu karstique, pour des occupations du Gravettien final
Figure 7 - Burins de Noailles provenant d'une occupation du Gravettien final (UE 4/UA 10) du site de Cardina I.

Figure 7 - Noailles burins recovered in a final Gravettian occupation (UE 4/UA 10) of Cardina I (Nila Nova Foz Côa). et terminal (Almeida 2003 ; Zilhão 1997 ; Almeida et al. 2004), que pour des phases plus anciennes dans des arènes granitiques conservées localement en plein air (Aubryet al. 2002 ; Aubry et Sampaio 2003a, 2003b), permettent ainsi une approche spatiale.

Sur le site sous-abri do Lagar Velho, qui occupe la rive orientée vers le nord d'une étroite vallée encaissée dans des calcaires crétacés, plusieurs phases d'occupation gravettiennes ont été définies, dont celles de la sépulture de l'enfant (cf. chapitre 1-5, Zihão et Trinkaus 2002). L'analyse spatiale des vestiges constituant le niveau d'occupation EE15 a permis de mettre en évidence la quasi absence de production laminaire et lamellaire sur l'assemblage d'un effectif de plus de 700 pièces, en association spatiale avec deux structures de combustion, dont l'une datée sur un fragment de charbon de Pinus sylvestris de $22493 \pm 107$ (Wk-9256). Les restes de faune chassée sont constitués par les os fragmentés des extrémités et des mandibules, dominés par le Cerf et le Bouquetin et, dans une moindre mesure, par le Cheval, le Sanglier, le Chamois et le Chevreuil. Plusieurs concentrations de vestiges lithiques correspondant à des séquences de débitage sur des galets de quartzite et blocs du silex local peuvent être mises en relation avec une des deux structures de combustion, de construction et fonctions distinctes et avec la préparation et la sélection de parties anatomiques charnues sur des carcasses obtenues lors de courts séjours de chasse (fig. 8, Zilhão et Almeida 2002 ; Almeida 2003). L'étude en cours des restes fauniques indiquerait la prédominance d'activités associées au traitement des peaux.

Dans un tout autre contexte géographique, l'analyse de l'organisation spatiale des vestiges lithiques seuls conser- 


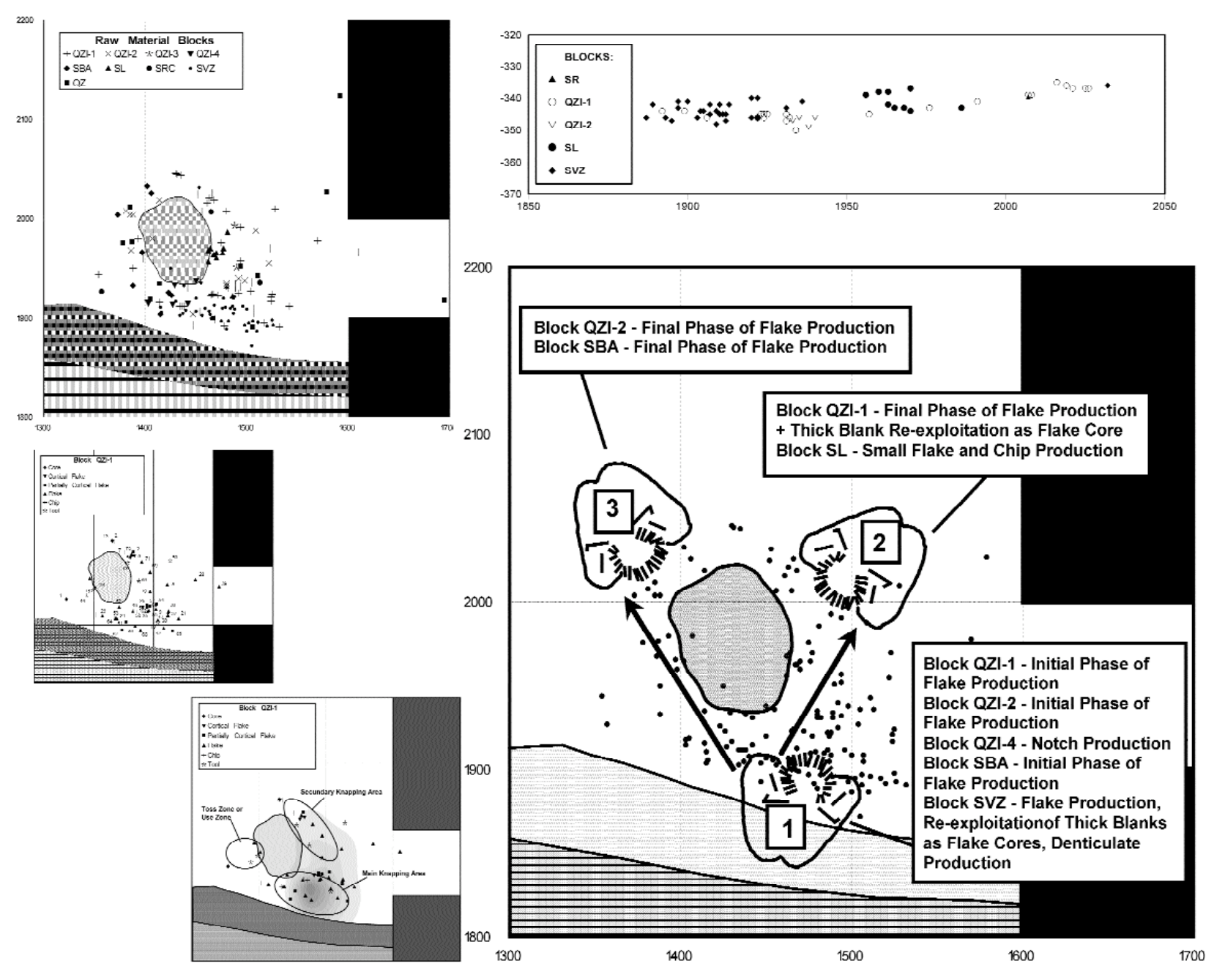

Figure 8 - Lagar Velho, analyse spatiale du niveau EE15 (QZ = variétés de quartz, S = variétés de silex).

Figure 8 - Lagar Velho. Spatial analysis of level EE15 (QZ = variety of quartz, S = variety of flint).

vés dans l'unité stratigraphique 3 du site de plein air de Olga Grande 4 (fig. $n^{\circ} 1$ ), a permis de mettre en évidence des séquences de réutilisation de blocs de quartz et de quartzite qui rentraient dans la constitution de structures de combustion de différentes morphologies et probablement de fonctions distinctes (fig. 9, Aubry et Sampaio 2003a, 2003b ; Aubry et al. 2003c). L'industrie lithique taillée, en relation avec ces structures, est (comme il a été noté pour l'occupation EE15 de l'abri de Lagar Velho) constituée d'éclats épais et larges obtenus sur des galets de quartzite et des blocs de quartz locaux et de supports lamellaires en silex et cristal, d'origine allochtone.
L'analyse de la répartition spatiale des différentes catégories de vestiges indique des déplacements et lieux de rejets distincts pour les éléments taillés et themo-fracturés, contraires au pendage des terrains, et une organisation spatiale qui indique une constitution pendant un même séjour, probablement de courte durée.

L'ensemble lithique découvert dans la couche $2 b$, du site de Lapa de Anecrial, cavité localisée à 340 mètres d'altitude, est associé à deux dates de $21560 \pm 680$ BP (ICEN964) sur charbon, par la méthode conventionnelle et de 21 $560 \pm 220$ BP (OxA-5526) par la méthode ASM sur un 


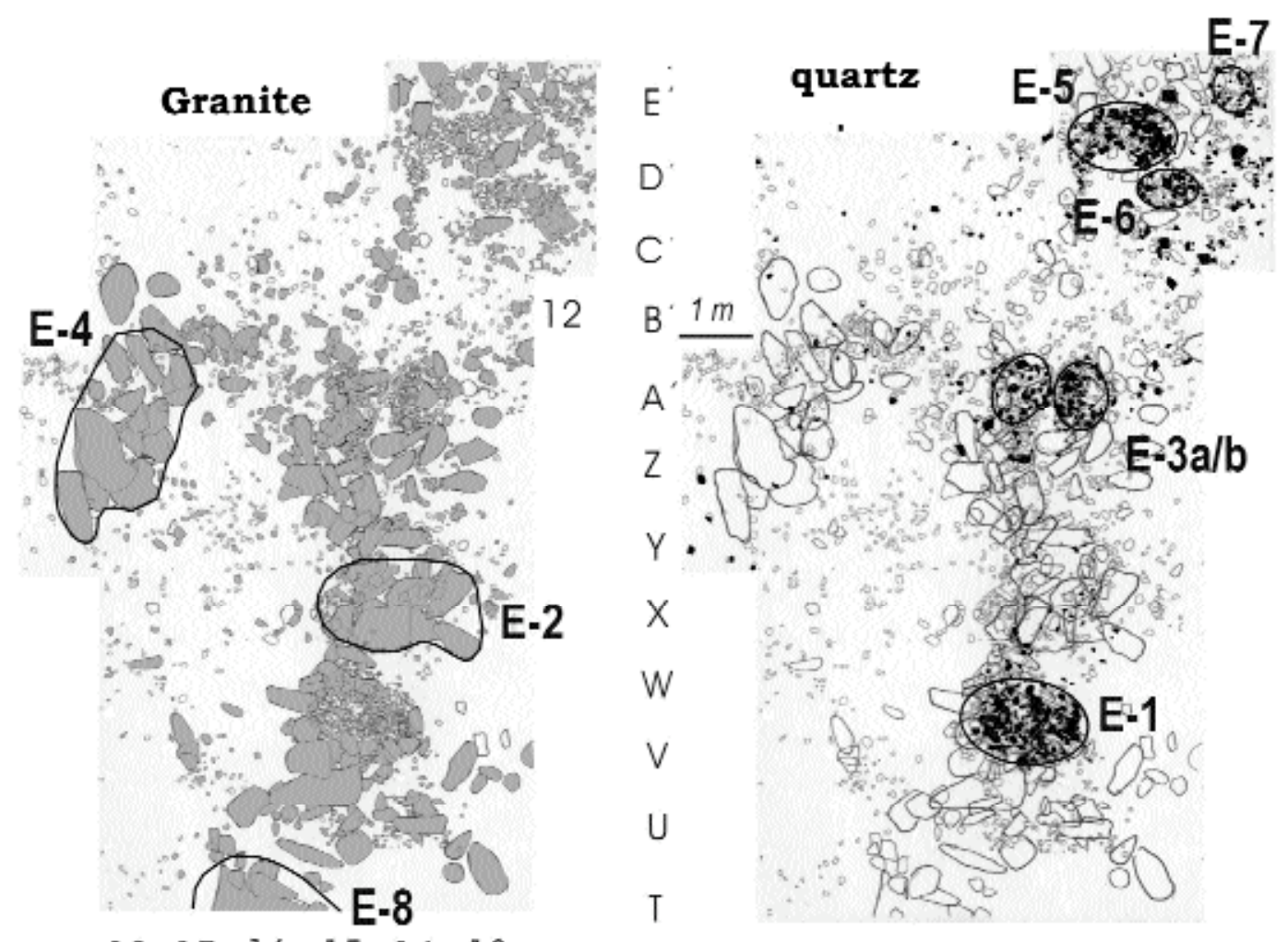

$\begin{array}{llllll}18 & 17 & 16 & 15 & 14 & 13\end{array}$

$\begin{array}{llllllll}19 & 18 & 17 & 16 & 15 & 14 & 13 & 12\end{array}$
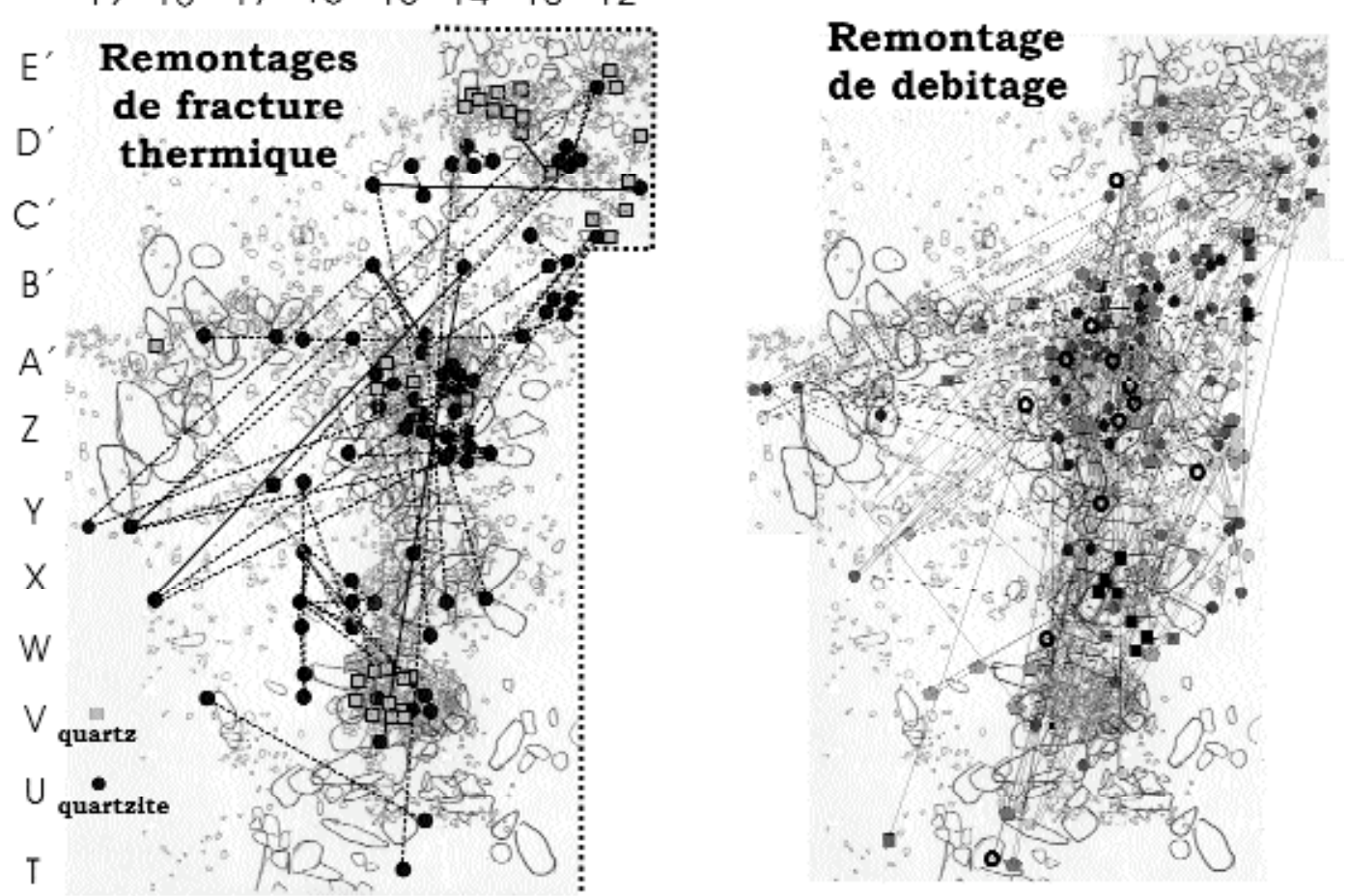

Figure 9 - Olga Grande 4 unité stratigraphique 3, répartition des éléments de plus de $5 \mathrm{~cm}$ et en quart, relations établies par remontage des éléments thermofracturés, en quartz et quartzite et remontage de l'industrie taillée.

Figure 9 - Olga Grande 4 level 3. Spatial distribution of $>5 \mathrm{~cm}$ items, distribution of quartz artefacts, refitting connections of quartz and quartzite thermoclasts, and stone tool refits. 

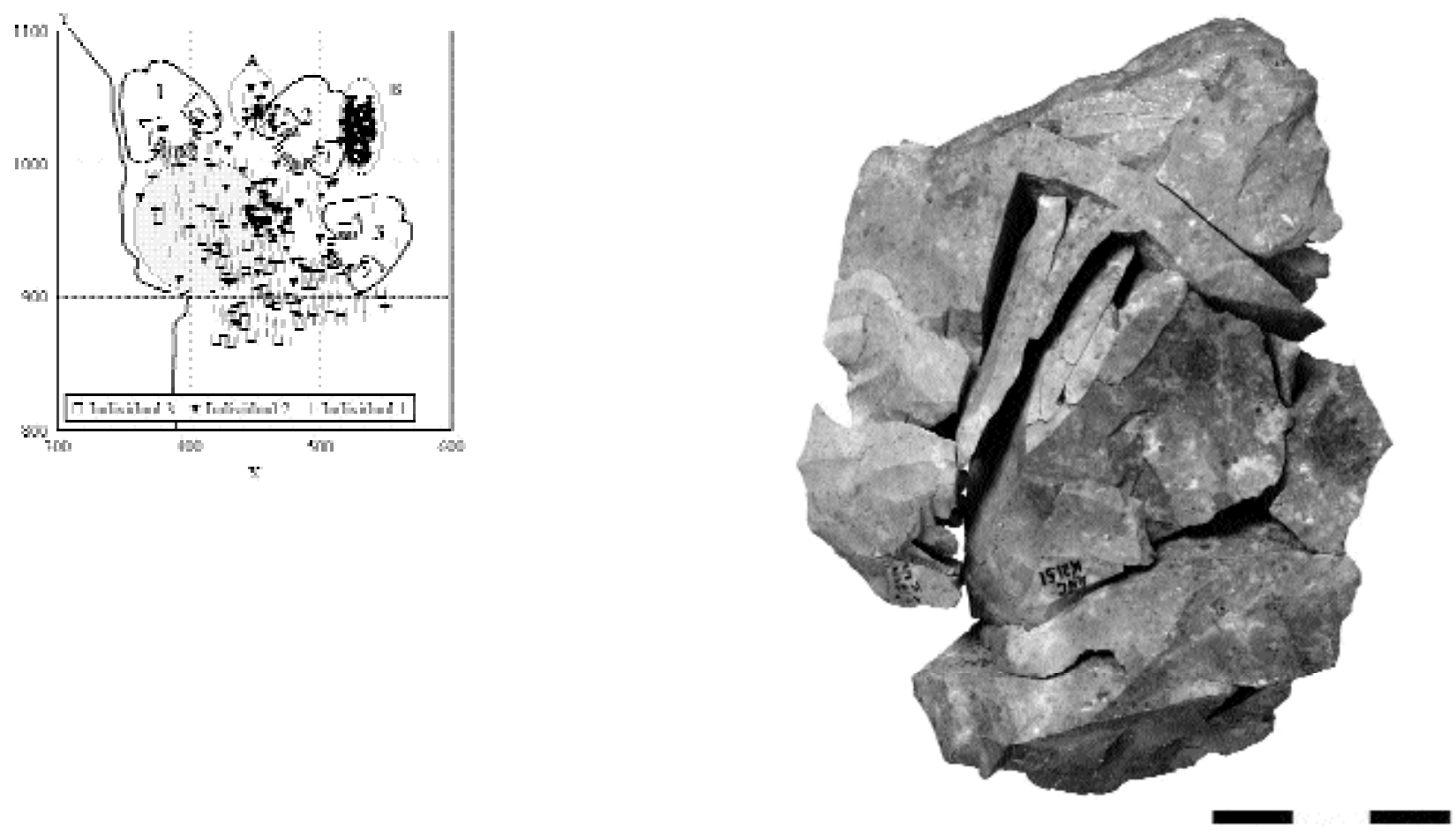

Figure 10 - Grotte de Anecrial, a : remontage d'un bloc de silex. b : analyse spatiale des vestiges lithiques de la couche 2.

Figure 10 - Anecrial. a: refitting flint nodule. b: spatial distribution of the lithics in level 2.

charbon d'Erica. Cette série a fait l'objet d'une analyse spatiale détaillée et d'une interprétation qui ont été exposées dans plusieurs articles (Zilhão 1997 ; Almeida 1998, 2000 ; Almeida et al. s.p, fig. 10a). Le remontage systématique des 586 vestiges lithiques de la couche 2 concerne $51 \%$, en effectif, et $92 \%$ du poids total (Almeida s.p.). II a permis de démontrer l'association sur une même plaquette, provenant d'une source de silex oxfordien distante de plus de $15 \mathrm{~km}$, de deux procédés de gestion et d'orientation des volumes de débitage ; prismatique sur une arête naturelle et de type grattoir caréné, sur des éclats retirés du même volume (fig. 10b). Ces derniers mettent en évidence un déficit en produits lamellaires, débités sur place, pour être probablement apportés ailleurs ou être montés (bruts ?) sur le fût d'une arme de chasse. L'analyse de l'organisation spatiale des vestiges lithiques et osseux (essentiellement des restes de lagomorphes) indique la constitution de cet assemblage archéologique à l'occasion du court séjour d'un groupe de quelques personnes. La répartition des vestiges correspond à l'utilisation d'un foyer en cuvette, probablement dans le cadre d'une halte intégrée dans une exploitation logistique des ressources de ce massif calcaire.

L'analyse des relations spatiales à une échelle géographique plus large, basée sur l'étude de la provenance des matières premières siliceuses, a vu un développement récent. Les travaux ont fourni les premières données permettant de mettre en évidence des déplacements pouvant dépasser deux cents kilomètres vers la vallée du Côa, une région sans ressource en silex (Aubry et Mangado 2003a, 2003b ; Aubry et al. 2001, 2004) ou bien vers l'extrémité occidentale du littoral de l'Algarve, aux ressources en silex de qualité médiocre (Bicho et al. 2004). Les modèles interprétatifs de déplacement de ces réserves de silex homogènes à grain fin (Cénomaniens de la région de Rio Maior, Leiria, Oxfordien de la vallée du Nabão, Bajocien/Bathonien de la région de Cantanhede), demandent encore à être testés. Ces premiers résultats demanderaient à être approfondis et fondés sur une meilleure connaissance et caractérisation pétrographique non seulement des sources de silex du Portugal, mais aussi de celles des formations miocènes du territoire espagnol limitrophe (vallée du Douro et ses affluents, vallée du Tage) car leur utilisation est attestée sur les sites de la vallée du Côa (Aubry et Mangado 2003a, 2003b ; Aubry et al. 2001, 2004 ; Bicho et al. 2004).

Ces résultats fournissent aussi des indications sur les secteurs qui ont été fréquentés pendant le Gravettien (et en général pendant tout le Paléolithique supérieur) où les indices archéologiques manquent encore (région de Salamanca, Ávila et Valladolid, en Espagne, régions d'Anadia et de Cantanhede, au Portugal). 


\section{4 - Environnement et mode de subsistance}

La reconstitution précise des paléo-environnements est encore trop peu avancée. Les travaux ponctuels effectués sur des assemblages fauniques associés à des industries gravettiennes (Aubry et al. 2001 ; Davis 2002 ; MorenoGarcía et Pimenta 2003 ; Bicho et al. 2003) ou des restes anthracologiques (Figueiral 1993 ; Figueiral et Terral 2002, Queiroz et al. 2002), permettent seulement de préciser le cadre paléoenvironnemental, établi sur la base des données océaniques ou des environnements morphosédimentaires et pédologiques de sites archéologiques (Angelucci 2002, 2003).

Les rares assemblages fauniques associés à des assemblages lithiques gravettiens étudiées (Cardoso 1992 ; Davis 2002 ; Aubry et al. 2001 ; Moreno-García et Pimenta 2003 ; Bicho et al. 2004) indiquent une rupture nette de la nature et des modalités d'accumulation des assemblages osseux avec les niveaux du Paléolithique moyen, sous-jacent, où l'accumulation semble surtout liée à la fréquentation par de grands camivores, en particulier la hyène (Crocuta sp.). Dans les assemblages associés à des niveaux d'occupation gravettiens, les traces de camivores existent toujours, surtout dans les assemblages à faible densité de vestiges du début de la séquence. Au sein des restes de faunes portant des traces anthropiques, les espèces détectées sont le Cerf, le Bouquetin, le Chamois, le Sanglier, le Cheval, l'Auroch et les Lagomorphes, ces derniers pouvant constituer une grande proportion de restes.

Les niveaux gravettiens d'occupations du site de Vale Boi, localisé à l'extrémité sud-ouest du Portugal (Algarve), distant de deux kilomètres de la côte atlantique actuelle, contiennent des restes de mollusques dont le milieu naturel actuel correspond à la zone intertidale (Bicho et al. 2004).

Les rares études anthracologiques effectuées sur les charbons récoltés dans des niveaux du Gravettien final, ont permis d'établir l'absence d'espèces méditerranéennes et de rapprocher les espèces présentes (Pynus sylvestris, Erica, Betula, Cytisus Scoparius, Ulex, Buxus sempervirens.., avec les assemblages actuels des reliefs calcicoles du versant sud des Pyrénées, à des altitudes comprises entre 1100 et 1800 mètres, où prédominent des paysages de végétation ouverte de type steppe (Queiroz et al. 2002 ; Figueiral in : Aubry et al. 2001).

\section{5 - Les restes humains}

Une seule sépulture gravettienne est connue en Péninsule ibérique. Elle a été découverte en 1998, au centre du Portugal, dans l'Abri du Lagar Velho (fig. 1 et 11). II s'agit d'un enfant mort dans sa cinquième année dont les restes ont été datés par le radiocarbone entre 24500 et 25000 BP (Zilhão et Trinkaus 2002). Le corps avait été déposé sur le dos, la tête légèrement inclinée sur le côté gauche, orientée vers l'est et les pieds vers l'ouest. Les bras étaient étendus le long du corps et la main droite reposait sur la hanche du même côté. Les jambes étaient légèrement fléchies et le bassin était un peu plus bas, témoignant, de même que la position légèrement plus élevée du crâne, d'une adaptation du corps à la morphologie en cuvette d'une fosse sépulcrale dont la profondeur peut être estimée entre 20 et $30 \mathrm{~cm}$. Sous les jambes, une nappe de charbons créée par la combustion d'une seule branche de pin sylvestre indique qu'un feu avait été allumé au fond de la fosse, préalablement à la déposition du corps.

Les ossements et les sédiments qui remplissaient l'espace vide avaient une intense couleur rouge. Le tout formait une tache aux limites nettes, concordant avec le contour du corps avant la décomposition des tissus mous. Cela indiquait que le cadavre avait du être enveloppé dans un "suaire " semi-rigide, probablement une peau d'animal. Près du cou, a été retrouvé un coquillage percé (Littorina obtusata), coloré lui aussi par l'ocre rouge ; un autre, cassé, fut récupéré dans le mince niveau de sédiments remaniés qui recouvrait le contexte funéraire en place et en faisait probablement aussi partie. A ces pendentifs qui faisaient peut-être partie d'un collier, il faut ajouter quatre canines de cerf percées, appartenant à quatre animaux différents, deux mâles adultes jeunes et deux femelles adultes âgées. L'analyse de leur procédé de perçage et leur étroite association spatiale avec les restes crâniens de l'enfant indiquent que ces objets devaient former une parure portée sur le front, arrangée de façon symétrique, les deux plus petits (des femelles) dans les extrémités et les deux plus grands (des mâles) au centre.

Une portion de colonne vertébrale et les côtes correspondantes d'un jeune lapin ont été découvertes sur les jambes. Cet ensemble appartenait sûrement à un squelette complet dont beaucoup d'autres éléments ont été retrouvés, eux aussi teints en rouge, dispersés à l'intérieur de la sépulture. II s'agit donc probablement d'une offrande funéraire correspondant à la déposition, sur le suaire enveloppant le corps d'un humain juvénile, du corps d'un lapin juvénile. Il est également fort probable que les deux bassins de cerf trouvés auprès des pieds et de l'épaule droite correspondent à des pièces de viande déposées près du corps de l'enfant, comme faisant partie du rituel funéraire, étant donné l'état de conservation de leur surface qui contraste avec l'altération marquée, causée par les camivores, des autres ossements trouvés dans les sédiments environnants.

Cette sépulture correspond à l'un des rares enterrements d'enfant de cette phase chronologique. II s'agit sans doute du plus complet et du mieux documenté que nous connaissions actuellement. Les détails du rituel funéraire et les caractéristiques des objets de parure pourraient indiquer une communauté de culture avec le sud-ouest de la France, l'Italie et l'Europe centrale.

\section{2- BILAN ET PERSPECTIVES}

\section{1- Les “ origines " du Gravettien}

Les " débuts " du Paléolithique supérieur au Portugal restent encore la zone la plus floue de la séquence chronostratigraphique puisque moins d'une dizaine de sites 


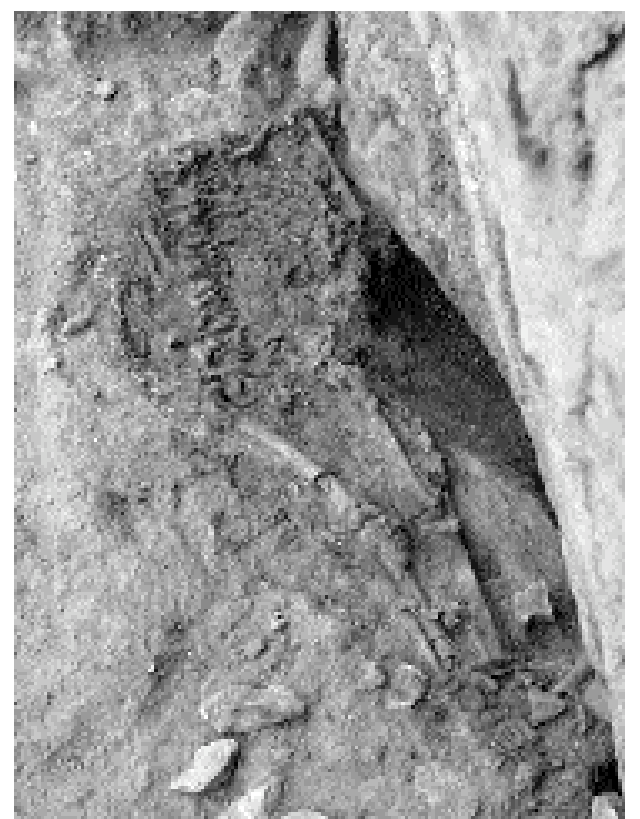

sont attribués à la période comprise entre 29000 et 25000 BP (Zilhão 1997 ; Bicho 2000 ; Thacker 2001 ; Aubry et al. 2006). Deux hypothèses ont été proposées pour expliquer cette faible densité, elle pourrait correspondre à une fréquentation éphémère par des populations modemes dans des territoires encore occupés par une population néandertalienne résiduelle (Marks 2000 ; Straus et al. 2000) ou serait la conséquence de la reprise, pendant plusieurs phases érosives, des sédiments qui seraient susceptibles de contenir les vestiges d'occupation contemporains de cette phase chronologique (Zilhão 2001).

L'étude géologique et les dates ASM obtenues pour la séquence de l'abri de Lagar Velho (Zilhão et Almeida 2003 ; Angelucci 2002, 2003) indiquent que la période antérieure à $27000 \mathrm{BP}$ est caractérisée par plusieurs discontinuités érosives majeures qui sont aussi observables sur plusieurs séquences karstiques, dans le Massif de Sicó contenant des vestiges d'occupation gravettienne (Aubry et al. 2006 ; Almeida et al. 2004). La comparaison avec la séquence observée dans la grotte de Buraca Escura (fig. 1 et 2) et la position stratigraphique des vestiges lithiques de ce gisement, indiquent une discontinuité érosive au sommet de l'ensemble 3 , sous-jacente à l'unité $2 \mathrm{f}$, qui contenait l'os de bouquetin en association avec un fragment distal de pointe à dos et daté par le procédé ASM de $26560 \pm 450$ BP. A cette pièce, est peut être associé un fragment mésial de lamelle à retouche alterne, que nous avions regroupé dans un article précédent (Aubry et al. 2001) avec les lamelles à dos tronqué du sommet de la couche 2e, mais qui se trouve en fait à la base de l'unité $2 \mathrm{~d}$, dans le remplissage d'une discontinuité érosive antérieure au dépôt de l'unité $2 e$, peut être contemporaine du dépôt de $2 f$ (fig. 2). Cette phase, ou ces phases érosives, peuvent être corrélées avec celles qui ont affecté les ensembles Bs et Al de l'abri de Lagar Velho (Angelucci 2002, 2003).
Figure 11 - Sépulture de l'enfant de l'abri de Lagar Velho.

Figure 11 - The Lagar Velho child burial.
Si les hypothèses d'attribution culturelle proposées ci-dessus sont correctes (fig. 5), de telles phases érosives furent aussi particulièrement marquées à l'Abri 1 de Vale dos Covões par des canaux érosifs affectant le sommet de l'ensemble 8o, interprétés par L. Dumiccio (Departamento de Ciências da Terra da Universidade de Coimbra) comme le résultat d'une concentration de l'écoulement d'une résurgence karstique située au fond de l'abri (fig. 5). Dans cette séquence, une telle phase peut donc être située, chronologiquement, entre l'occupation caractérisée par des lamelles appointées à retouche alterne et des lamelles à dos à la base du niveau $8 \mathrm{~b}$, et l'occupation gravettienne à pointes à dos des unités 6 et 7, elle-même surmontée par des occupations caractérisées par des microgravettes (Aubryet al. 2006).

Dans la proposition de Zilhão (1997), les lamelles Dufour confectionnées par retouche alterne sur des supports lamellaires à profil rectiligne seraient contemporaines du site de plein air de Vale de Porcos, où des lamelles de morphologie semblable ont été produites sur des nucléus carénés et de type burin des Vachons, en association avec une production laminaire de grand module (par contraste avec les assemblages lithiques de tout le Paléolithique supérieur du Portugal). À la grotte de Pego do Diabo, des lamelles Dufour sont associées à plusieurs dates dont une de $28120+820-760$ BP (ICEN-732) obtenue sur des esquilles d'os.

La comparaison de ces éléments avec les armatures classées dans les deux catégories typologiques des fléchettes et des gravettes provenant de la base de la couche 5 de l'Abri Pataud (Bricker 1995, p. 54 et 55) révèle l'association de types très distincts. Des lamelles étroites appointées par retouche alterne, dont certaines sont semblables aux exemplaires du site de Pego do Diabo (Zilhão 1997) sont associées à des pointes à dos, obtenues par une retouche croisée. Si l'attribution que nous proposons est confimée 
par le résultat de dates sur d'autres matériaux que les charbons (fig. 5), la série provenant de la base de la couche $8 \mathrm{~b}$ de Vale dos Covões présente une association semblable. Néanmoins, la rareté et la disparité des informations technologiques et typologiques disponibles pour les phases récentes de l'Aurignacien et en particulier pour les assemblages dominés par les burins carénés et les burins des Vachons, aussi bien que l'absence d'analyse taphonomique pour la couche 5 de l'Abri Pataud permettant de définir la relation entre les différentes unités (avant/arrière, supérieur/moyen/inférieur), la variabilité, des différentes catégories typologiques d'armatures sur lamelle des phases récentes de l'Aurignacien et des phases " moyen-ancien " du Gravettien, reste à établir.

Quoi qu'il en soit, la nature des séquences litho-stratigraphiques et archéologiques et l'existence d'une phase érosive majeure font, par conséquent, que les données disponibles au Portugal, ne nous semblent pas encore suffisantes pour reconstituer de façon sûre les modalités de fréquentation de ces régions et la signification des changements des outillages lithiques pendant la fourchette chronologique qui s'étend entre 30000 et 25000 BP.

\section{2 - Bilan et perspectives de recherche}

Si les stades évolutifs qui mènent du Gravettien final au Protosolutréen semblent maintenant bien établis et calés chronologiquement (Zilhão 1997 ; Almeida 2000 ; Aubry et al. 2001 ; Zilhão et Almeida 2003), les données disponibles pour les phases antérieures montrent bien le double impact des dynamiques sédimentaires dans la destruction des matériaux organiques permettant l'obtention de datations absolues et pouvant occasionner des remaniements post-dépositionnels des vestiges lithiques.

Les sites qui ont fait l'objet d'une analyse technologique et spatiale par le biais de remontages lithiques montrent, aussi bien pour les vestiges d'occupation en milieu karstique (Aubry et al. 2001 ; Almeida 2003) que pour ceux provenant des plateaux granitiques de l'intérieur (Aubry et Sampaio 2003a, 2003b), que les vestiges ont été abandonnés lors d'occupations courtes. Les activités documentées sont majoritairement axées sur le traitement des carcasses et la réparation des parties lithiques des armes de chasse, ou bien liées directement à la présence de silex et à la production de réserves de supports laminaires, d'outils ou de nucléus, en vue d'une utilisation différée. L'étude technique en cours par L. Klaric (bourse post-doctorale Fyssen), des méthodes de production des supports lamellaires et des techniques de retouche des pointes à dos et lamelles à dos des différentes phases individualisées jusqu'à présent, aussi bien que le développement d'études tracéologiques (M. Araújo), pourraient permettre de tester l'interprétation fonctionnelle des productions d'éclats en quartz et en quartzite basée sur l'analyse de l'organisation spatiale de ces productions.

La relation entre la production d'esquilles et de lamelles par percussion posée sur enclume et la confection d'armes de chasse reste encore à établir pour permettre une approche technique globale de la production lithique et l'interprétation fonctionnelle des sites. On peut espérer que la mise en évidence de stigmates d'impact, sur des supports lamellaires ou des esquilles non retouchées, parallèlement à la constitution d'un référentiel expérimental de montage de ces produits et de fractures lors de l'utilisation sur des projectiles en matériaux organiques, apportera de nouveaux arguments pour expliquer la faible proportion des armatures à dos, ou même leur absence, pendant certaines phases et dans certains sites gravettiens du Portugal.

Les niveaux du Gravettien final de Cabeço de Porto Marinho et de Terra do Manuel, dans le bassin de Rio Maior, correspondent probablement à des occupations plus longues, à caractère résidentiel, comme c'est le cas aussi pour les sites de l'énigmatique Fontesantense. À Cabeço de Porto Marinho, cependant, les fouilles n'ont pu affecter qu'une portion périphérique des occupations, coupées par une sablière et à Terra do Manuel les fouilles modernes n'ont pu exploiter qu'une petite zone marginale épargnée par les fouilles anciennes. Seuls les niveaux d'occupation du Gravettien final du site de Cardina I (Vila Nova de Foz Côa) sont donc suffisamment intacts et denses pour que les assemblages lithiques et les structures conservées puissent être interprétés comme correspondant à des occupations résidentielles (Aubry et al. 2003).

Ce déficit en sites résidentiels peut être interprété comme la conséquence d'un biais dans la conservation ou la détection des indices constituant notre registre archéologique. Afin de pallier ces manques, plusieurs orientations peuvent être proposées :

- la prospection systématique des plateaux occupant les points les plus hauts des massifs calcaires de l'Estremadura où, selon le modèle établi pour la vallée du Côa (Aubry et al. 2002), la préservation d'un couvert neigeux pendant le Pléniglaciaire supérieur aurait pu être à l'origine d'accumulations saisonnières d'eau qui pourraient avoir attiré les grands herbivores ;

- la prospection systématique et la réalisation de sondages dans les dépôts alluviaux des bassins versants où les précipitations sont collectées sur de faibles superficies pourraient permettre de mettre en évidence des vestiges d'occupations humaines antérieures au Dryas III, conservés au contact entre les versants et la plaine alluviale ;

- la prospection systématique et suivie de la bande littorale actuelle des secteurs où des dépôts antérieurs au Pléniglaciaire supérieur se sont conservés et sont observables malgré la couverture éolienne accumulée pendant l'Holocène, tel que ceux qui contiennent des troncs de Pinus sylvestris détectés et datés entre $20700 \pm 300$ BP et $29100 \pm 510 \mathrm{BP}$, au sud de Espinho, entre les plages de Esmoriz, Cortegaça et Maceda (Granja et al. 1996). 


\section{BIBLIOGRAPHIE}

ALMEIDA F. 1998 - O método das remontagens líticas: enquadramento teórico et aplicações. Trabalhos de Arqueologia da E.A.M., Lisboa, 3, p. 1-40.

ALMEIDA F. 2000 - The Te rminal Gravettien of Portuguese Estremadura. Ph. D. Dissertation. Southern Methodist University.

ALMEIDA F. 2003 - Paleotecnologia no Abrigo do Lagar Velho (Leiria): contribuição do método das remontagens líticas para o estudo tecnológico e paleoetnográfico de uma ocupação gravettense. In: MATEUS, J. E. ; MORENOGARCÍA, M., ed. - Paleoecologia Humana e Arqueociências: Um programa multidisciplinar para a arqueologia sob a tutela da cultura. Lisboa: Instituto Português de Arqueologia (Trabalhos de Arqueologia; 29) p. 317-324.

ALMEIDA F., s.p. - Reffiting at Lapa Do Anecrial: Studying Technology and Micro Scale Spatial Patterning Through Lithic Reconstructions. In: FITTING ROCKS. Lithic Refitting Examined. Edited by Utsav Schurmans and Marc De Bie.

ALMEIDA F., BRUGAL J.-Ph. et ZILHÃO J. 2004 - An Upper Paleolithic Pompeii: Technology, Subsistence and Paleoethnography at Lapa do Anecrial, Comunicação ao Congresso de Faro.

ALMEIDA M., NEVES M.J. 2003 - Ocupação holocénica do Vale das Buracas (Zambujal, Condeixa-a-Nova, Coimbra), in: Estudos Pré-históricos, Vila Nova de Paiva (Portugal): Centro de Estudos Pré-históricos da Beira Alta.

ALMEIDA F., ANGELUCCI D., GAMEIRO C., CORREIA J., PEREIRA T. 2004 - Novos dados para o Paleolítico Superior final da Estremadura Portuguesa: resultados preliminares dos trabalhos arqueológicos de 1997-2003 na Lapa dos Coelhos (Casais Martanes, Torres Novas). Promontoria, Faro, 2 :2, p.157-192.

ALMEIDA F, GAMEIRO C., ZILHÃO J. 2002 - The artifact Assemblages. In :

ZILHÃO J., TRINKAUS E. (eds) (2002) - Portrait of the artist as a Child. The Gravettian Human Skeleton from the Abrigo do Lagar Velho and its Archaeological Context. Trabalhos de Arqueologia, 22, Instituto Português de Arqueologia, Lisboa., p. 202-219.

ALMEIDA M, NEVES M.J., CUNHA L., DIMUCCIO L. et AUBRY T. 2004 - Contributo da sequência cultural Pleistocénico-Holocénico parar a compreensão da génese e evolução do canhão flúvio-cársico do Vale das Buracas. Programa e resumos do $2^{\circ}$ Congresso Nacional de Geomorfologia, Coimbra, 11-13 Novembro de 2004. p. 39.

ALMEIDA M., DIMUCCIO L., A., AUBRY T., NEVES M.J. et CUNHA L.- s.p. Enquadramento geomorfológico e cronocultural do sítio arqueológico da Gândara do Outil I. In:
Actas do $2^{\circ}$ Congresso Nacional de geomorfologia, Coimbra, 11-13 Novembro.

ANGELLUCCI D.E. 2002 - The Lagar Velho rock-shelter (lapedo, Leiria, Portugal): stratigraphic record and palaeoenvironnement during the Oxygen Isotope stage 2. Publicações da Associação portuguesa de geomorfologia, Vol. 1, Contribuições para a Dinâmica geomorfológica, Associação Portuguesa de Geomorfologia, Lisboa, 2002.

ANGELLUCCI D.E. 2002 - The Geoarchaological Context. In: Portrait of the Artist as a Child. Zilhão (J.), Trinkaus (E.) (eds) (2003) - Portrait of the artist as a Child. The Gravettian Human Skeleton from the Abrigo do Lagar Velho and its Archaeological Context. Trabalhos de Arqueologia, 22, Instituto Português de Arqueologia, Lisboa, p. 58-91.

AUBRY T. 1998 - Olga Grande 4: uma sequência do Paleolítico superior no planalto entre o Rio Côa e a Ribeira de Aguiar. Revista Portuguesa de Arqueologia. Lisboa. 1:1, p. 5-26.

AUBRY T. 2001 - L'occupation de la basse vallée du Côa pendant le Paléolithique supérieur. In ZILHÃO, J., AUBRY T., CARVALHO A. F., ed. - Les premiers hommes modernes de la péninsule ibérique (Actes du Colloque de la Commission VIII de l'UISPP. Vila Nova de Foz Côa, 2224 Octobre 1998). Lisboa: IPA, p. 253-273.

AUBRY T. 2002 - Le contexte archéologique de l'art paléolithique à l'air libre de la vallée du Côa. In SACCHI, D., ed. - L'art paléolithique à l'air libre: le paysage modifié par l'image (Tautavel, Campôme, 7-9 octobre 1999). SaintEstève: GAEP; GÉOPRE, p. 25-38.

AUBRY T., SAMPAIO J.D. 2003a - O Método das remontagens de vestígios líticos: aplicação ao nível de ocupação gravettense do sítio de Olga Grande 14 (Almendra, Vila Nova de Foz Côa). In: Paleoecologia Humana e Arqueociências, Um Programa Multidisciplinar para a Arqueologia sob a Tutela da Cultura. MATEUS, J.E. e MORENO-GARCIA M. eds. TRABALHOS DE ARQUEOLOGIA 29,: 327-330.

AUBRY T., SAMPAIO J.D. 2003b - Remontagem de rochas termo-alteradas ; um meio de reconstrução dos modos de funcionamento de estructuras de combustão no sítio de Olga grande 4 (Almendra, Vila Nova de Foz Côa), In: Paleoecologia Humana e Arqueociências, Um Programa Multidisciplinar para a Arqueologia sob a Tutela da Cultura. MATEUS, J.E. e MORENO-GARCIA M. eds. TRABALHOS DE ARQUEOLOGIA 29, : 331-335.

AUBRY T., ALMEIDA M., NEVES M.-J. 2006 - The Middleto-Upper Paleolithic transition in Portugal : An Aurignacian phase or not? Proceeding of the Symposium «Towards a definition of the Aurignacian", held in Lisbon, Portugal, June 25-30, 2002, O. BAR-YOSEF e J. ZILHAO (eds). Trabalhos de Arqueologia ; 45 : 95-108.

AUBRY T., BRUGAL J.P., CHAUVIÈRE F.X., FIGUEIRAL I., MOURA M.H. et PLISSON H. 2001 - Modalités d'occupa- 
tion au Paléolithique supérieur dans la grotte de Buraca Escura (Redinha, Pombal, Portugal). Revista Portuguesa de Arqueologia, Volume 4, n², 2001, p. 19 - 46.

AUBRY T., MANGADO LLACH J. 2003a - Modalidades de aprovisionamento em matérias-primas líticas nos sítios do Paleolítico Superior do Vale do Côa: dos dados à interpretação. In MATEUS, J. E. ; MORENO-GARCÍA, M., ed. Paleoecologia Humana e Arqueociências: Um programa multidisciplinar para a arqueologia sob a tutela da cultura. Lisboa: Instituto Português de Arqueologia (Trabalhos de Arqueologia; 29), p. 340-342.

AUBRY T., MANGADO LLACH X. 2003b - Interprétation de l'approvisionnement en matières premières siliceuses sur les sites du Paléolithique supérieur de la vallée du Côa (Portugal). In Les matières premières lithiques en Préhistoire (table ronde internationale organisée à Aurillac, Cantal, du 20 au 22 juin 2002). Carcassonne: Association Préhistoire du Sud-Ouest (Prehistoire du Sud-Ouest; Supplément $n^{\circ} 5$ ), p. 27-40.

AUBRY T., CHAUVIERE F.X., MANGADO LLACH X. et SAMPAIO J.D. 2003c - Constitution, territoires d'approvisonnement et fonction des sites du Paléolithique supérieur de la basse vallée du Côa. In: BAR S1122 2003: Perceived Landscapes and Built Environments. The cultural geography of Late Paleolithic Eurasia. Acts of the XIVth UISPP Congress, University of Liège, Belgium, 2-8 September 2001. Colloques / Symposia $6.2 \& 6.5$ edited by S. A. Vasil'ev, O. Soffer and J. Kozlowski.

AUBRY T., MANGADO LLACH X., FULLOLA J. M., ROSELL L. et SAMPAIO J. D. 2004 - Raw Material Procurement in the Upper Paleolithic Settlements of the Côa Valley (Portugal): New Data Concerning Modes of Resource Exploitation in Iberia. In Abstract. 6.th annual meeting of the EEA, Lisbon 10-17 September 2000.

AUBRY T., MANGADO LLACH X., SAMPAIO J. D. et SELLAMI F. 2002 - Open-Air Rock-Art and Modes of Exploitation during the Upper Paleolithic in the Côa Valley (Portugal). Antiquity, York. 76, p. 62-76.

AUBRY T., GAMEIRO C., KLARIC L. (en préparation) L'abri de Vale dos Covões (Soure, Portugal) : apport à la chronologie et au peuplement de l'occident de la Péninsule ibérique pendant le Paléolithique supérieur.

BICHO N.F. 2000 - Revisão crítica dos conhecimentos actuais do Paleolítico superior português. Actas do II Congresso de Arqueologia Peninsular (Vila Real, 1999) Porto, p. 425-442.

BICHO N.F., STINER M., LINDLY J., REID FERRING C. et CORREIA J. 2004- Preliminary Results from the Upper Paleolithic Site if Vale Boi, Southwestern Portugal. Joumal of Iberian Archaeology, vol.5, 2003, p. 51-66.

BICHO N.F, HAWS J., HOCKETT B., MARKOVA A. et BELCHER W. 2003 - Paleoecologia e occupação humana da
Lapa do Picareiro: resultados preliminares. REVISTA PORTUGUESA DE ARQUEOLOGIA, Vol. 6, n², p-49-81.

BRICKER H.M. Dir. 1995 - Le Paléolithique supérieur de l'abri Pataud (Dordogne) : les fouilles de H.L. Movius Jr. : suivi d'un inventaire analytique des sites aurignaciens et périgordiens de Dordogne. Paris, Ed. de la Maison des Sciences de l'Homme, 1995. 328 p. ; ill. (DAF ; 50).

CARDOSO J. 1992 - Contribuição para o conhecimento dos grande mamíferosdo Plistocénico Superior de Portugal. Thèse de Doctorat de l'Université de Lisbonne.

DAVIS S. 2002 - The mammals and birds from the Gruta do Caldeirão, Portugal. Revista Portuguesa de Arqueologia. Lisboa. 5:2, p. 29-98.

FIGUEIRAL I. 1993 - Cabeço de Porto Marinho : une approche paléoécologique. Premiers résultats. In: Fumanal, M.P., Bernabeu J. (eds.), Estúdios sobre Quatemario. Médios sedimentários, câmbios ambientales, habitat humano. University Valência, Valência, p. 176-172.

FIGUEIRAL I. et TERRAL J.F. 2002 - Late Qutemary refugia of mediterranean taxa in the Portuguese Estremadura : charcoal based palaeovegetation and climatic reconstruction. Quatemary Science Reviews, 21 (2002), p. 549-558.

GOUTAS N. 2004 - Caractérisation et évolution du Gravettien en France par l'approche techno-économique des industries en matières dures animales (étude de six gisements du Sud-ouest). Thèse de Doctorat en Préhistoire -Ethnologie-Anthropologie de l'Université de Paris I, 2 Volumes, 675 p.

GRANJA H.M., SOARES DE CARVALHO, G., DE GROOT T., de MONGE SOARES A. et PARISH R. 1996 Geochronology and the Recent Geomorphological Evolution of the Northwest Zone of Portugal. Partnership in Costal Zone Management, J. Taussik \& J. Mitchell (eds), 1996, Samara Publishing Limited, Cardigan.

KLARIC L. 2000 - Note sur la présence de lames aménagées par technique de Kostienki dans les couches gravettiennes du Blot (Cerzat,Haute-Loire), BSPF, tome 97, $\mathrm{n}^{\circ} 4$, pp. 625-636.

KLARIC L. "2003 - L'unité technique des industrie à burins du Raysse dans leur contexte diachronique. Réflexions sur la diversité culturelle au Gravettien à partir des données de la Picardie, D'Arcy-sur-Cure, de Brassempouy et du Cirque de la Patrie., Thèse de Doctorat de Préhistoire de l'Université de Paris I, 426 p.

MARKS A.-E. 2000 - Upper Paleolithic occupation of Portugal: resident vs. visitors. Actas do III Congresso de Arqueologia Peninsular (Vila Real, 1999). Porto, 2: 341-349.

MARKS A.-E. et ALMEIDA F. 1996 - The late Aurignacian and "Aurignacian" Elements in the Upper 
Palaeolithic of the Portuguese Estremadura, Portugal. 6. The Upper Palaeolithic, Colloquium XI and Colloquium XII, XIII International Congress of Prehistoric and Protohistoric Sciences, Forli, 8/14 September 1996, : 11-21.

MERCIER N., VALLADAS H., FROGET L., JORONS J.L., REYSS J.L. et AUBRY T. 2001 - Application de la méthode de la thermoluminescence à la datation des occupations paléolithiques de la vallée du Côa.In : les premiers hommes modernes de la Péninsule ibérique. Vila Nova de Foz Côa, 1998 (Trabalhos de Arqueologia, 17), p. 275-280.

MORENO-GARCÍA M. et PIMENTA C.M. 2002 - The Paleofaunal Context. In : Zilhão J., Trinkaus E. (eds) (2003) - Portrait of the artist as a Child. The Gravettian Human Skeleton from the Abrigo do Lagar Velho and its Archaeological Context. Trabalhos de Arqueologia, 22, Instituto Português de Arqueologia, Lisboa, p. 112-131.

PALMA DI CESNOLA A. 1996 - Le Gravettien le plus ancien en Italie. In: 6. The Upper Palaeolithic, Colloquium $\mathrm{XI}$ and Colloquium XII, XIII International Congress of Prehistoric and Protohistoric Sciences, Forlì, 8/14 September 1996 : p.227-235.

PETIT P. B., VAN DER PLICHT H., RAMSEY C. B., MONGES SOARES A. et ZILHÃO J. - The radiocarbon Chronology. In : Zilhão (J.), Trinkaus (E.) (eds) (2003) Portrait of the artist as a Child. The Gravettian Human Skeleton from the Abrigo do Lagar Velho and its Archaeological Context. Trabalhos de Arqueologia, 22, Instituto Português de Arqueologia, Lisboa, p. 132-138.

QUEIROZ P., VAN LEEUWAARDEN W. et MATEUS J. 2002 - The Paleovegetational Context, in ZILHÃO, J.; TRINKAUS, E. (eds.) - "Portrait of the Artist as a Child. The Gravettian Human Skeleton from the Abrigo do Lagar Velho and its Archeological Context", Trabalhos de Arqueologia 22, Lisboa, Instituto Português de Arqueologia, p. 92-111.

ROCHE J. 1951 - Le niveau Paléolithique supérieur de la Grotte de Casa da Moura (Cesareda). Communicações dos Serviços Geológicos de Portugal., 32, p.103-122

ROCHE J., FRANCA J.C., FERREIRA O.V. et ZBYSZEWSKI G. 1962 - Le Paléolithique supérieur de la grotte de Salemas (Ponte de Lousa). Communicações dos Seniços Geológicos de Portugal, 46, p. 197-207.

SACCHI D., SOLER MASFERRER S., MAROTO GENOVER J. et DOMENECH FAUS E. 1996 - La question de l'Aurignacien tardif dans le domaine méditerranéen nordoccidental. In : 6. The Upper Palaeolithic, Colloquium XI and Colloquium XII, XIII International Congress of Prehistoric and Protohistoric Sciences, Forli, 8/14 September 1996, p. 23-40.

STRAUS L.-G., BICHO N., WINEGARDNER A.-C. 2000 The Upper Paleolithic settlement of lberia: first generation maps. Antiquity, : 553-566.
THACKER P. 1996 - Hunter-gatherer lithic economy and settlement systems: understanding regional assemblage variability in the Upper Paleolithic of Portuguese Estremadura. In ODELL, G. eds., Stone Tools: Theorical Insights into Human Prehistory. New York; Plenum Presss, p.1001-124.

THACKER P. 2001 - The aurignacian campsite at Chainça, and its relevance for the earliest Upper Palaeolithic settlement of the Rio Maior vicinity. Revista Portuguesa de Arqueologia, 4 (1), : 5-15.

VALLADAS H., MERCIER N., FROGET L., JORONS J.L., REYSS J.L., AUBRY T. 2001 - TL dating of Upper Palaeolithic sites in the Côa Valley (Portugal). Quatemary Science Reviews. 20:5-9, p 939-943.

ZILHÃO J. 1991a - Portugal (1986-1990). In: Le Paléolithique supérieur européen. Rapport Quinquennal 1986-1991, Union intemationale des sciences préhistoriques et protohistoriques. Commission VIII : Etudes et Recherches Archéologiques de L'Université de Liège 52, p. 315-319.

ZILHÃO J. 1991b - Aurignacien et Gravettien au Portugal. In : Actes du XII ${ }^{\circ}$ Congrès International des Sciences Préhistoriques et Protohistoriques, Aurignacien en Europe et au Proche Orient. Bratislava, 1-7 septembre 1991. Institut Archéologique de l'Académie Slovaque des Sciences. p.154-162.

ZILHÃO J. 1996 - Le Paléolithique supérieur du Portugal. Bilan quinquennal 1991-1995. In : Le Paléolithique supérieur européen, Bilan Quinquennal 1991-1996, Union internationale des sciences préhistoriques et protohistoriques. Commission VIII : Etudes et Recherches Archéologiques de L'Université de Liège 76, p. 369-380.

ZILHÃO J. 1997a - O Paleolítico superior da Estremadura portuguesa, 2 Volumes, Lisboa, Ed. Colibri.

ZILHÃO J. 1997b (coord) - Arte rupestree Pré-Historia do Vale do Côa. Trabalhos 1995-1996. Relatório Científico ao Govemo da República portuguesa elaborado nos termos da resoluçao do Conselho de Ministros n 4/96, de 17 de Janeiro.

ZILHÃO J. 2000 - Nature and culture in Portugal from 30,000 to $20,000 \mathrm{bp}$, in ROEBROEKS, W.; MUSSI, M.; SVOBODA, J.; FENNEMA, K. (eds.) - "Hunters of the Golden Age: The Mid Upper Palaeolithic of Eurasia 30,000 20,000 BP", Leiden, University of Leiden, p. 337354.

ZILHÃO J. 2001 - Le Paléolithique supérieur du Portugal. Bilan Quinquennal 1997-2001. In : Le Paléolithique supérieur européen, Bilan Quinquennal 1996-2001, Commission VIII - XIV ${ }^{\circ}$ Congrès UISPP (Liège, 2-8 septembre 2001, Lièges, Etudes et Recherches Archéologiques de L'Université de Liège 97, p. 161-171.

ZILHÃO J. et ALMEIDA F. 2002 - The Archaeological Framework, in ZILHÃO, J.; TRINKAUS, E. (eds.) - 
"Portrait of the Artist as a Child. The Gravettian Human Skeleton from the Abrigo do Lagar Velho and its Archeological Context", Trabalhos de Arqueologia 22, Lisboa, Instituto Português de Arqueologia, p. 29-57.

ZILHÃO J., MARKS A. E., FERRING C. R., BICHO N. F. et FIGUEIRAL I. 1995 - The Upper Paleolithic of the Rio Maior basin (Portugal). Peliminary results of a 1987-1993 PortugueseAmerican research project. "Trabalhos de Antropologia e Etnologia”, 35 (4), Porto, p. 69-88.
ZILHÃO J. et TRINKAUS E. (eds) (2002) - Portrait of the artist as a Child. The Gravettian Human Skeleton from the Abrigo do Lagar Velho and its Archaeological Context. Trabalhos de Arqueologia, 22, Instituto Português de Arqueologia, Lisboa. 